\title{
Biomaterial-Based Nanocomposite for Osteogenic Repurposing of Doxycycline
}

This article was published in the following Dove Press journal:

International Journal of Nanomedicine

\author{
Salma El-Habashy (D) \\ Hoda Eltaher (D) \\ Ahmed Gaballah (iD ${ }^{2}$ \\ Radwa Mehanna (iD) 3,4 \\ Amal H El-Kamel (iD) \\ 'Department of Pharmaceutics, Faculty of \\ Pharmacy, Alexandria University, \\ Alexandria, 21521 , Egypt; ${ }^{2}$ Microbiology \\ Department, Medical Research Institute, \\ Alexandria University, Alexandria, 2156I, \\ Egypt; ${ }^{3}$ Medical Physiology Department, \\ Faculty of Medicine, Alexandria \\ University, Alexandria, 2II3I, Egypt; \\ ${ }^{4}$ Center of Excellence for Research in \\ Regenerative Medicine and Applications \\ CERRMA, Faculty of Medicine, \\ Alexandria University, Alexandria, 21 I3I, \\ Egypt
}

Background: Besides its antimicrobial action, doxycycline (DX) has lately been repurposed as a small-molecule drug for osteogenic purposes. However, osteogenic DX application is impeded by its dose-dependent cytotoxicity. Further, high-dose DX impairs cell differentiation and mineralization.

Purpose: Integrating DX into a biomaterial-based delivery system that can control its release would not only ameliorate its cytotoxic actions but also augment its osteogenic activity. In this work, we managed to engineer novel composite DX-hydroxyapatite-polycaprolactone nanoparticles (DX/HAp/PCL) to modify DX osteogenic potential.

Methods: Employing a $2^{3}$-factorial design, we first optimized HApN for surface-area attributes to maximize DX loading. Composite DX/HAp/PCL were then realized using a simple emulsification technique, characterized using various in vitro methods, and evaluated for in vitro osteogenesis.

Results: The developed HApN exhibited a favorable crystalline structure, $\mathrm{Ca}: \mathrm{P}$ elemental ratio (1.67), mesoporous nature, and large surface area. DX/HAp/PCL achieved the highest reported entrapment efficiency $(94.77 \% \pm 1.23 \%)$ of DX in PCL-based particles. The developed composite system achieved controlled release of the water-soluble DX over 24 days. Moreover, the novel composite nanosystem managed to significantly ameliorate DX cytotoxicity on bone-marrow stem cells, as well as enhance its overall proliferation potential. Alkaline phosphatase and mineralization assays revealed superior osteodifferentiation potential of the composite system. Quantification of gene expression demonstrated that while DX solution was able to drive bonemarrow stem cells down the osteogenic lineage into immature osteoblasts after 10-day culture, the innovative composite system allowed maturation of osteodifferentiated cells. To the best of our knowledge, this is the first work to elaborate the impact of DX on the expression of osteogenic genes: RUNX2, OSP, and BSP. Further, the osteogenicity of a DX-loaded particulate-delivery system has not been previously investigated.

Conclusion: Our findings indicate that repurposing low-dose DX in complementary biomaterialbased nanosystems can offer a prominent osteogenic candidate for bone-regeneration purposes.

Keywords: bone regeneration, bioactivity, mesenchymal stem cells, drug repositioning, osteodifferentiation

\section{Introduction}

Bone is a dynamic tissue of structural complexity. Bone extracellular matrix (ECM) comprises inorganic calcium phosphate nanocrystals embedded in an unmineralized organic collagen matrix. ${ }^{1}$ Under the umbrella of regenerative medicine, boneregeneration research is an impactful multidisciplinary field that employs a versatile range of biomaterials and therapeutic moieties for the purposes of bone regeneration. $^{2}$
Correspondence: Amal H El-Kamel Department of Pharmaceutics, Faculty of Pharmacy, Alexandria University, I Khartoum Square, Azarita, PO Box 21521 , Alexandria, Egypt Tel +20-10-0508 0510

Email amalelkamel@yahoo.com
International Journal of Nanomedicine 2021:16 I I03-I I26 
Protein therapeutics have long been used to promote bone regeneration: various growth factors promote cell differentiation and proliferation in vitro. ${ }^{3}$ For example, bone morphogenic proteins (BMPs) have been the most widely used biologicals for treating orthopedic disorders. ${ }^{4}$ However, this approach is associated with numerous shortcomings in vivo, with unwarranted longterm safety and efficacy. ${ }^{3}$ The use of BMP2 has been associated with swelling, ectopic bone formation, and adjacent bone resorption. ${ }^{5}$ Drawbacks of using growth factors generally encompass adverse immunogenic reactions, challenging manufacturing techniques, and compromised product stability, which all hinder wide translational application of growth factors in the clinical setting. ${ }^{5}$ In this respect, the advent of small molecularweight drug molecules has offered the possibility to overcome various limitations of protein therapeutics. They are easier to manufacture at lower cost, provide more stability, and exert lower immunogenic action, and hence can be used to reversibly regulate specific functions of physiological protein growth factors. ${ }^{5}$ Since some of these small molecules are already US Food and Drug Administration (FDA)-approved for use in other health conditions in human, the repurposing of these drugs for bone-therapy applications can provide efficient candidates with well-documented biological behavior. ${ }^{4}$

Among these small molecules, the repurposed antibiotic doxycycline (DX) has been linked to promising osteogenic activity that is not related to its antimicrobial action. Lowdose DX exerts osteoinductive actions similar to BMP2 on osteoprogenitor cells, ${ }^{6}$ implying DX can be advocated as a valuable small molecular-weight alternative. Although the underlying mechanism has not yet been fully elucidated, reports have suggested that it acts by inhibiting osteoclast formation and inducing osteoclast apoptosis. ${ }^{7}$ Other studies refer osteogenic DX actions to inhibiting collagenase activity. ${ }^{8}$ Even though low-dose DX is emerging as a promising osteogenic candidate, higher doses have shown deleterious side effects in vitro. ${ }^{6,9,10}$ For instance, unlike lower-dose DX, a $100 \mu \mathrm{M}$ DX dose exerts cytotoxic actions and negative impacts on the mineralization and differentiation of osteoprecursor cells. $^{10}$

Various inorganic biomaterials have been involved in bone-regeneration applications. In this regard, hydroxyapatite nanoparticles $(\mathrm{HApN})$ are very popular. ${ }^{11}$ With great chemical proximity to the bone mineral $\mathrm{HAp}\left(\mathrm{Ca}_{10}\left[\mathrm{PO}_{4}\right]_{6}\right.$ $\left.[\mathrm{OH}]_{2}\right)$, HApN can be directly involved in osteoprogenitor-cell differentiation and mineralization and stand out as a great osteoconductive and bioactive material. ${ }^{11}$ Numerous studies have addressed the application of HAp-based systems for osteogenic purposes. ${ }^{12-14}$ For example, composite chitosan-based scaffolds with magnesium-doped HAp have provided a biocompatible substrate for stem-cell proliferation and differentiation. ${ }^{12}$

Another commonly applied class of materials are biodegradable polymers, with polycaprolactone (PCL) being one of the most popular. ${ }^{1}$ PCL is a semicrystalline synthetic biodegradable polymer that is physiologically biocompatible, ${ }^{15}$ and gained the FDA approval in the 1980s. ${ }^{15}$ PCL-based systems have achieved favorable results as controlled-release delivery systems for boneregeneration purposes, ${ }^{16}$ as well as plain substrates with osteogenic potential. ${ }^{17}$ For example, Kamath et al ${ }^{16}$ managed to control resveratrol release from albumin nanoparticles by further embedding them into PCL scaffolds, which also exhibited enhanced mineralization of mesenchymal stem cells.

From this perspective, we hypothesized that integrating DX within a composite-delivery system containing HApN and PCL could provide controlled release of the active agent over time. As such, this composite system would limit direct DX contact with cells, eliminate its dose-dependent cytotoxicity, and augment its repurposed osteogenic potential. Although several studies have investigated the osteogenic potential of low-dose DX solution on cells in vitro, ${ }^{6,8,9,18}$ the osteogenic action of a composite DX-loaded particulate-delivery system has not yet been addressed. Therefore, in this study, we extrapolated from our previous work establishing the osteogenic potential of hybrid HAp-PCL nanoparticles, ${ }^{19}$ in order to osteogenically repurpose DX using a nanocomposite-delivery system. The novel DX-loaded nanocomposite managed to provide controlled release of DX for prominent osteogenicity via lowering dose-dependent DX cytotoxicity, as well as the integration of bioactive components of complementary osteogenic potential. First, HApN were prepared using wet chemical precipitation and optimized for their surface-area properties to maximize DX loading using a $2^{3}$ factorial design. DX-loaded HApN were further embedded in a PCL matrix, and this novel nanocomposite was evaluated using bone marrow-derived mesenchymal stem cells (BMSCs) under osteoinduction conditions using various techniques. 


\section{Methods}

\section{Materials}

DX hydrochloride was a gift from the European Egyptian Pharmaceutical Industries, Alexandria, Egypt, and dexamethasone sodium phosphate was a gift from Pharco Pharmaceutical, Alexandria, Egypt. Dichloromethane (DCM), dimethyl sulfoxide (DMSO), and polyvinyl alcohol (PVA; MW $14 \mathrm{kDa}$ ) were purchased from El-Nasr Pharmaceutical, Cairo, Egypt. Calcium chloride dihydrate $\left(\mathrm{CaCl}_{2}\right), \mathrm{L}$-ascorbic acid, and sodium- $\beta$-glycerophosphate pentahydrate were obtained from Loba Chemie, Mumbai, India. PCL (MW $80 \mathrm{kDa}$ ) was purchased from Birmingham Polymers, Birmingham, AL, USA. Tris-HCl was purchased from Promega, Madison, WI, USA. A calcium reagent kit was obtained from NS Biotec, Alexandria, Egypt, and an alkaline phosphatase-assay kit was acquired from Spectrum Diagnostics, Cairo, Egypt. COSMO cDNA-synthesis and Hera SYBR green qPCR kits were purchased from Willowfort, Birmingham, UK. TRI reagent was obtained from Zymo Research. Triton X100 was purchased from PerkinElmer. MTT was obtained from Serva, Heidelberg, Germany. Low-glucose $(1 \mathrm{~g} / \mathrm{L})$ DMEM) and penicillin-streptomycin $(10 \mathrm{IU} / \mathrm{mL} / 10 \mu \mathrm{g} /$ $\mathrm{mL}$ ) were purchased from Lonza, Basel, Switzerland. Bradford reagents (Sigma-Aldrich, St Louis, MO, USA), FBS (HyClone, GE Healthcare), alizarin red S (Oxford Lab Fine Chem, Maharashtra, India), BSA (Biowest, Nuaillé, France), and disodium hydrogen orthophosphate anhydrous $\left(\mathrm{Na}_{2} \mathrm{HPO}_{4}\right.$; Winlab) were used in this study.

\section{Preparation of HApN}

HApN were prepared via wet precipitation. ${ }^{19,20}$ Precursor solutions of $\mathrm{CaCl}_{2}$ and $\mathrm{Na}_{2} \mathrm{HPO}_{4}$ were prepared in deionized water with a molar $\mathrm{Ca}: \mathrm{P}$ ratio of 1.67 , as per standard HAp stoichiometry, ${ }^{20}$ and $\mathrm{pH}$ was adjusted to $10 \pm 0.5$. HApN chemical precipitation progressed by the dropwise addition of $\mathrm{PO}_{4}{ }^{3-}$ ion solution to $\mathrm{Ca}^{2+}$ ion solution under magnetic stirring (Eurostar; IKA Labortechnik, Staufen im Breisgau, Germany). Precipitation proceeded at $50^{\circ} \mathrm{C}$ while maintaining $\mathrm{pH}$ at $11-12$ for higher-purity HAp. ${ }^{21}$ This was followed by aging of the prepared particles. Afterward, the mixture was centrifuged (3K-30; Sigma, Osterode, Germany) at $15,000 \mathrm{rpm}, 4^{\circ} \mathrm{C}$ for 15 minutes to separate the formed pellet, which was washed three times with water, then absolute ethanol.

For optimization of HApN and elaboration of the impact of different process parameters on surface-area properties, a two- level $\left(2^{3}\right)$ factorial design was employed. HApN formulations were prepared by varying precursor concentrations, aging time, and aging temperature. Design-Expert (version 9.0.6.1; Stat-Ease, Minneapolis, MN, USA) was used for establishing the design model and further data analysis.

\section{Preparation of Doxycycline- Hydroxyapatite Nanoparticles (DX/HAp)} DX loading was carried out as previously reported, ${ }^{22}$ with slight modifications. HApN were dispersed by sonication (digital bath sonifier; Elma, Singen, Germany) in DX ethanolic solution of different concentrations and the homogeneous dispersion was stirred overnight. The whole dispersion was then centrifuged at $15,000 \mathrm{rpm}, 4^{\circ}$ C for 15 minutes to separate the formed pellet.

\section{Preparation of Composite Doxycycline- Hydroxyapatite-Polycaprolactone Nanoparticles (DX/HAp/PCL)}

DX/HAp/PCL were prepared using S/O/W emulsion-solvent evaporation. ${ }^{23}$ The previously separated DX/HAp pellet was dispersed in PCL solution in DCM of different concentrations and the mixture was homogenized (Ultra Turrax; IKA Labortechnik, Staufen, Germany) in the aqueous solution containing $0.5 \%$ PVA with or without $\mathrm{NaCl}$, followed by probe sonication for 5 minutes (Sonoplus HD 3100; Bandelin Electronics, Berlin, Germany). The ratio of organic phase to aqueous phase was set to 1:10. For particle solidification, organic solvent evaporation proceeded by stirring under vacuum for 2 hours. The formed particles were separated by centrifugation at $10,000 \mathrm{rpm}$, $25^{\circ} \mathrm{C}$ for 10 minutes.

For comparative analysis, DX/PCL particles were also prepared using $\mathrm{W}_{1} / \mathrm{O} / \mathrm{W}_{2}$ emulsion-solvent evaporation. Briefly, DX solution (2\%) in 1\% PVA was emulsified in the organic polymer solution in DCM using probe sonication. The mixture was then homogenized in the aqueous solution $(0.5 \% \mathrm{PVA}$ and $4 \% \mathrm{NaCl}$ in deionized water). Further processing was carried out as previously mentioned for DX/ HAp/PCL. For further studies on HApN, DX/HAp, or composite DX/HAp/PCL, freeze-dried samples (LyoQuest; Telstar, Terrassa, Spain) or dispersions were used.

\section{Characterization Methods} Surface-Area Analysis

Nitrogen-adsorption and -desorption isotherms were obtained (Belsorp Mini II analyzer; BEL, Osaka, Japan). 
Freeze-dried HApN samples were processed by degassing at $150^{\circ} \mathrm{C}$ for 3 hours, followed by nitrogen adsorption. Brunauer-Emmett-Teller (BET) and Barrett-JoinerHalenda analyses were employed for surface-area and pore-size studies.

\section{Scanning Electron Microscopy-Energy-Dispersive X- Ray (SEM-EDX) Analysis}

Scanning electron microscopy (SEM; JSM-IT200; Jeol, Tokyo, Japan) coupled with EDX analysis was used to investigate the elemental composition of the prepared HApN. Ca:P ratio was averaged from three separate measurements.

\section{X-Ray Diffraction (XRD)}

The crystallinity of HAp was characterized using X-ray diffraction (XRD; D2 Phaser; Bruker, Madison, WI, USA). A copper source (wavelength $1.54184 \mathrm{~A}^{\circ}$ ) was used at $30 \mathrm{kV}$ voltage and $10 \mathrm{~mA}$ current. Samples were scanned over the $2 \theta$ range: $10^{\circ}-80^{\circ}$ at $0.01^{\circ}$ step size and 0.05 seconds step time.

\section{Transmission Electron Microscopy (TEM)}

Field-emission transmission electron microscopy was employed (JEM-2100F; Jeol, Tokyo, Japan) for morphology and particle-size analysis. Particle dispersions were mounted on copper grids, dried prior to examination, and $4 \%$ uranyl acetate staining was applied for composite DX/HAp/PCL. For particle-size analysis, 50 measurements were conducted with microscopy using image-analysis software (Fiji 1.52p; National Institutes of Health, Bethesda, MD, USA). ${ }^{24}$

\section{Fourier-Transform Infrared Spectroscopy (FTIR)} FTIR spectroscopy was conducted (Cary 630; Agilent Technologies, USA) to investigate chemical interactions among ingredients. Samples were scanned over 4,000-650 $\mathrm{cm}^{-1}$ at $2 \mathrm{~cm}^{-1}$ resolution.

\section{Determination of Drug Content}

For direct determination of DX-entrapment efficiency (EE) in DX/HAp, composite DX/HAp/PCL, and DX/ PCL, pellets obtained after centrifugation were dissolved in $1 \mathrm{~N} \mathrm{HCl}, \mathrm{DMSO}-1 \mathrm{~N} \mathrm{HCl}$ and DMSO, respectively. DX concentration in the obtained solutions was then determined by spectroscopy (UV-1700; Shimadzu, Kyoto, Japan) at $346 \mathrm{~nm}^{23}$ DX EE was calculated using Equation 1:

$$
\begin{aligned}
\text { Entrapment efficiency } \%= & \frac{\text { Actual drug amount in pellet }}{\text { Initial drug amount added }} \\
& \times 100 \%
\end{aligned}
$$

Drug loading in DX/HAp was calculated using Equation 2:

$$
Q=\frac{W_{D}}{W_{C}}
$$

where $\mathrm{Q}(\mathrm{mg} / \mathrm{mg})$ is the amount of drug loaded per unit weight of $\mathrm{HApN}, \mathrm{W}_{\mathrm{D}}(\mathrm{mg})$ the drug amount practically determined in the DX/HAp pellet, and $\mathrm{W}_{\mathrm{C}}(\mathrm{mg})$ the weight of the carrier HApN used. To investigate the DX-adsorption mechanism, experimental drug-loading data were fitted to both the Langmuir and Freundlich models. Both models are often used to reveal adsorption kinetics. ${ }^{25}$

\section{In Vitro Drug Release}

Dispersion of DX/HAp and composite DX/HAp/PCL in water was used. Aliquots corresponding to $1 \mathrm{mg}$ DX were used for drug release in $10 \mathrm{~mL}$ purified water. ${ }^{26}$ Release experiments were carried out in a shaking water bath at $37^{\circ} \mathrm{C}$ and $50 \mathrm{rpm}$ (Wisebath; Daihan Scientific, Seoul, South Korea). After different time intervals, samples were aliquoted for spectroscopic analysis.

\section{Determination of Hydroxyapatite}

\section{Content}

HAp content was determined via calcium colorimetric analysis using a calcium-reagent kit according to the manufacturer's instructions. ${ }^{27}$ HAp was dissolved using $1 \mathrm{~N}$ $\mathrm{HCl}$ and calcium ion in the extracts determined. Reaction yield for HApN was calculated as the ratio of the practical to the theoretical reaction yield. Also, incorporation for HApN was set as the actual to the initial amount of HApN used to prepare DX/HAp and composite DX/HAp/PCL formulations.

\section{In Vitro Cell-Culture Studies}

Experiments in these studies were performed at the Center of Excellence for Research in Regenerative Medicine Applications, Alexandria Faculty of Medicine (AFM), Alexandria, Egypt. Animal handling was in accordance with the guidelines of the International Council of Laboratory Animal Science and approved by the Research Ethics Committee, AFM (00012098). Briefly, BMSCs isolation was performed by applying direct adhesion $^{28}$ using male Sprague Dawley albino rats. 
Femur and tibia samples were used for cell extraction from $\mathrm{BM}$, and cells were cultured in complete culture medium (CCM) containing FBS (10\% v:v), L-glutamine (1\% v:v), and penicillin-streptomycin (1\% v:v) in low-glucose DMEM. Cells were maintained at $37^{\circ} \mathrm{C}$ in a humidified incubator at $5 \% \mathrm{CO}_{2}$, with constant medium replacement every 2-3 days. Regular cell monitoring was performed via phase-contrast microscopy (CKX41; Olympus, Tokyo, Japan), and passages 3-5 were used for all experiments.

\section{Cell Viability and Proliferation}

MTT assays were used for assessment of cell viability. BMSCs cultured at $5 \times 10^{3}$ cells/well in 96-well plates were left to adhere for 24 hours, then divided into different groups receiving plain CCM (control) and DX solution, DX/HAp, and composite DX/HAp/PCL suspensions in CCM (corresponding to 50 and $100 \mu \mathrm{g} / \mathrm{mL} \mathrm{DX)} \mathrm{and}$ incubated for 48 hours. Then, MTT solution $(0.5 \mathrm{mg} / \mathrm{mL}$ in CCM) was added after medium removal, incubated with cells for 4 hours, then replaced with DMSO. Optical density (OD) were spectroscopically obtained at $570 \mathrm{~nm}$ (ELX 800; BioTek, California, USA) and cell-viability percentage was calculated as any group OD to the OD of the control (CCM) group.

\section{Alkaline Phosphatase (ALP)-Activity Assay}

BMSCs cultured in CCM at $1.5 \times 10^{4}$ cells $/ \mathrm{cm}^{2}$ in 12 -well plates were divided into groups after reaching $70 \%$ confluence. Different groups received CCM (control), osteogenic differentiation medium (ODM), DX solution, or formulations corresponding to $50 \mu \mathrm{g} / \mathrm{mL}$ DX in ODM (treatment groups). ODM comprised DMEM, $50 \mu \mathrm{g} / \mathrm{mL}$ ascorbic acid, $10^{-4} \mathrm{mM}$ dexamethasone and $10 \mathrm{mM} \beta$ glycerophosphate. ${ }^{29}$ ALP activity was assessed after culture for 5 and 10 days, where cells were hydrolyzed in lysis buffer $(0.2 \%$ Triton X-100 in $1 \mathrm{M}$ Tris-HCl, $\mathrm{pH} 7.4)$ by freezing then thawing for one cycle then sonication at $4^{\circ} \mathrm{C}$ for 15 seconds. ${ }^{29}$ The obtained cell lysates after centrifugation were used for ALP spectroscopy determination using an ALP-assay kit. Total protein content was also quantified using Bradford's reagent applying a linearized Bradford assay. ${ }^{30}$ ALP activity was expressed as $\mu$ mole enzyme/minute/mg total protein.

\section{Alizarin Red S Mineralization Assay}

Mineralized deposits in the ECM were quantified using alizarin red S staining. ${ }^{29}$ BMSCs were cultured in six-well plates as previously stated for ALP assays. Alizarin red S staining was conducted for all groups after 5-, 10-, and 15- day culture. After discarding of culture media and thorough washing of cells using PBS, fixation was carried out using $10 \%$ formaldehyde for 10 minutes. Then, cells were washed using PBS and stained for 15 minutes using $2 \% \mathrm{w}$ : $\mathrm{v}$ alizarin red $\mathrm{S}$ solution of $\mathrm{pH}$ 4.2. Stained calcium nodules were quantified by image analysis of at least 10 photomicrographs using Fiji image-analysis software. ${ }^{24}$

\section{Quantitative Real-Time Polymerase Chain Reaction (qRT-PCR) Analysis}

In order to elaborate the molecular-level impact of the applied formulations, qRT-PCR was employed to quantify the expression of osteogenesis-related genes ${ }^{13}$ for all groups after 10-day culture. Lineage-specific genes were tested, namely RUNX2, OSP, and BSP. GAPDH was used as the housekeeping gene (primer sequences in Table 1). BMSCs cultured in 12-well plates were used as previously mentioned for ALP assays. mRNA was extracted using TRI reagent according to the manufacturer's protocol, then quantified (DS-11 FX; DeNovix, Delaware, USA). Reverse transcription was then carried out using a cDNA-synthesis kit according to the manufacturer's protocol, and a thermocycler (Veriti; Applied Biosystems, California, USA). Real-time PCR was conducted on a Stratagene Mx 3000 (Applied Biosystems, California, USA) using a qPCR kit following the manufacturer's protocol. Afterward, the $2^{-\Delta \Delta \mathrm{Ct}}$ method for quantitative data analysis was applied and lineage-specific gene foldchange obtained by normalizing the expression of said gene to GAPDH expression.

\section{Statistics}

At least three replicates were obtained for most experiments, and means \pm SD are used for data expression. ANOVA followed by Duncan's post hoc pairwise

Table I Primer Sequences used for qRT-PCR

\begin{tabular}{|l|l|l|}
\hline Gene & \multicolumn{2}{|l|}{ Sequence (f, forward; $\boldsymbol{r}$, reverse) } \\
\hline RUNX2 & F & $\begin{array}{l}\text { CCCCATCCATCCATTCCACC } \\
\text { GGTGGCAGTGTCATCATCTGAA }\end{array}$ \\
\hline RSP & F & $\begin{array}{l}\text { GCCACACTCTCAGGGGTAAC } \\
\text { CTCCAGCCTTCTTGGGTAGC }\end{array}$ \\
\hline R P & F & $\begin{array}{l}\text { CACAGTCGATGTCCCTGACG } \\
\text { GTGGCATCGGGATACTGTTCA }\end{array}$ \\
\hline GAPDH & R & $\begin{array}{l}\text { TGAAGCTCATTTCCTGGTATGACA } \\
\text { TGGGTGGTCCAGGGTTTCTT }\end{array}$ \\
\hline
\end{tabular}


comparison tests were applied for statistical analysis using SPSS. $p \leq 0.05$ was considered statistically significant unless otherwise mentioned.

\section{Results and Discussion}

\section{Preparation and Optimization of HApN}

The most popular technique for synthesis of HApN is wet chemical precipitation. ${ }^{21}$ One precursor is mixed with the other in solution in different adjustable synthesis conditions with variable impact on particle architecture and surface area. ${ }^{21}$ Nanoparticles in general have the advantage of high surface area and hence reactivity. ${ }^{31}$ More specifically, HAp in the nanoform exhibits higher surface area than larger HAp particles, and this inherent property greatly impacts HApN reactivity and adsorption ability. In consequence, such adsorptive capacity guides the use of $\mathrm{HApN}$ as drug-delivery device, as well as interaction with physiological proteins and thus bioactivity. ${ }^{13}$

In this study, we used a $2^{3}$ factorial design to investigate the effect of initial precursor concentration (A), aging time (B), and aging temperature (C) on HApN surface-area attributes (Table 2). To the best of our knowledge, this is the first research in literature to study these variables and effects. Mean pore diameter (MPD, nm), total specific surface area $\left(\mathrm{a}_{\mathrm{s}, \mathrm{BET}}, \mathrm{m}^{2} / \mathrm{g}\right)$ and total pore volume $\left(\mathrm{V}_{\mathrm{P}}\right.$, $\mathrm{cm}^{3} / \mathrm{g}$ ) were used as responses in the developed design.

\section{Investigation of HApN Surface Area}

Tailoring surface area and pore diameter of HApN can be attained by changing various synthesis parameters. In this study, we prepared eight formulations using wet chemical precipitation for HApN optimization (Table 2). MPD, $\mathrm{a}_{\mathrm{s}, \mathrm{BET}}$, and $\mathrm{V}_{\mathrm{P}}$ were $11.29 \pm 0.30-16.59 \pm 0.52 \mathrm{~nm}, 124.53 \pm 3.05-$ $204.00 \pm 1.44 \mathrm{~m}^{2} / \mathrm{g}$, and $0.51 \pm 0.02-0.84 \pm 0.01 \mathrm{~cm}^{3} / \mathrm{g}$, respectively. It is important to note that the precursor concentration (A) is denoted by calcium molar concentration to simplify data processing and presentation, with a fixed Ca:P ratio of 1.67.

Nitrogen-adsorption and -desorption isotherms obtained for all formulations exhibited the characteristic pattern of typeIV isotherms according to International Union of Pure and Applied Chemistry classification (representative F6 isotherm shown in Figure 1A). For typeIV isotherms, gas adsorption on mesoporous materials proceeds via multilayer adsorption, followed by capillary condensation. The slight curvature at the beginning of the curve represents the stage where multilayer adsorption begins. On the other hand, the hysteresis loop in the high relative pressure $\left(\mathrm{P} / \mathrm{P}_{0}\right)$ range of $0.8-1$ is
Table 2 Independent Variables and Responses Applied in the Factorial Design for Hydroxyapatite Nanoparticle Optimization

\begin{tabular}{|c|c|c|c|c|c|c|}
\hline \multirow[t]{2}{*}{$\begin{array}{l}\text { Test } \\
\text { code }\end{array}$} & \multicolumn{3}{|c|}{$\begin{array}{c}\text { Experimental } \\
\text { variables }\end{array}$} & \multicolumn{3}{|c|}{ Responses } \\
\hline & $\begin{array}{c}\text { A } \\
(\mathrm{M})\end{array}$ & $\begin{array}{c}\text { B } \\
\text { (hour) }\end{array}$ & $\mathrm{C}\left({ }^{\circ} \mathrm{C}\right)$ & $\begin{array}{l}\text { MPD } \\
(\mathrm{nm})\end{array}$ & $\begin{array}{l}a_{\mathrm{s}, \mathrm{BET}} \\
\left(\mathrm{m}^{2} / \mathrm{g}\right)\end{array}$ & $\begin{array}{c}V_{P} \\
\left(\mathrm{~cm}^{3} / g\right)\end{array}$ \\
\hline \multirow[t]{2}{*}{$\mathrm{FI}$} & 0.1 & 24 & 50 & 16.59 & 124.53 & 0.51 \\
\hline & & & & \pm 0.52 & \pm 3.05 & \pm 0.029 \\
\hline \multirow[t]{2}{*}{ F2 } & 0.1 & 24 & 25 & 12.73 & 163.82 & 0.52 \\
\hline & & & & \pm 0.50 & \pm 2.41 & \pm 0.028 \\
\hline \multirow[t]{2}{*}{ F3 } & 0.06 & 1 & 50 & 13.00 & I58.64 & 0.60 \\
\hline & & & & $\pm 0.5 \mathrm{I}$ & \pm 9.85 & \pm 0.001 \\
\hline \multirow[t]{2}{*}{$\mathrm{F} 4$} & 0.1 & I & 50 & 12.70 & $|86.4|$ & 0.77 \\
\hline & & & & \pm 0.01 & \pm 0.02 & \pm 0.007 \\
\hline \multirow[t]{2}{*}{ F5 } & 0.1 & I & 25 & $|3.7|$ & 137.36 & 0.61 \\
\hline & & & & \pm 0.02 & \pm 8.12 & \pm 0.001 \\
\hline \multirow[t]{2}{*}{ F6 } & 0.06 & I & 25 & 16.28 & 199.34 & 0.84 \\
\hline & & & & \pm 0.55 & \pm 9.66 & \pm 0.013 \\
\hline \multirow[t]{2}{*}{ F7 } & 0.06 & 24 & 50 & 16.39 & 156.35 & 0.59 \\
\hline & & & & \pm 0.14 & \pm 8.59 & \pm 0.032 \\
\hline \multirow[t]{2}{*}{ F8 } & 0.06 & 24 & 25 & 11.29 & 204.00 & 0.57 \\
\hline & & & & \pm 0.30 & $\pm \mathrm{I} .44$ & \pm 0.011 \\
\hline
\end{tabular}

Notes: A, precursor concentration; $B$, aging time; $C$, aging temperature.

Abbreviations: MPD, mean pore diameter; $a_{\mathrm{s}, \mathrm{BET}}$, total specific surface area (Brunauer-Emmett-Teller); $V_{B}$ total pore volume.

associated with capillary condensation. The H1-hysteresis loop indicates cylindrical pore geometry, relatively high pore-size uniformity and pore connectivity. ${ }^{32}$ Pore size-distribution plots were obtained using the BarrettJoiner-Halenda method, and calculations were conducted on the desorption branch of the isotherm. Poresize distribution for the eight formulations tested confirmed the presence of mesoporous materials (average pore size $14.09 \pm 2.04 \mathrm{~nm}$, representative F6 plot shown in inset of Figure 1A). In general, mesoporous nanomaterials stand out as efficient drug-delivery systems. Increasing surface area and pore volume provide an anchor for drug loading and subsequent modulated release. $^{33}$

Within the range of conditions used in this study, all factors examined in the design were shown to influence all three responses, namely mean pore diameter $(\mathrm{nm})$, total specific surface area $\left(\mathrm{m}^{2} / \mathrm{g}\right)$, and total pore volume $\left(\mathrm{cm}^{3} / \mathrm{g}\right)$. ANOVA (Table 3 ) revealed the models for all responses to be significant $(p \leq 0.001)$ with proper predictiveness and goodness of fit, as indicated by determination coefficient $\left(R^{2}\right)$. It was identified from regression equations that different factors exerted interaction effects over all responses. Generally, the presence of interactions among studied 


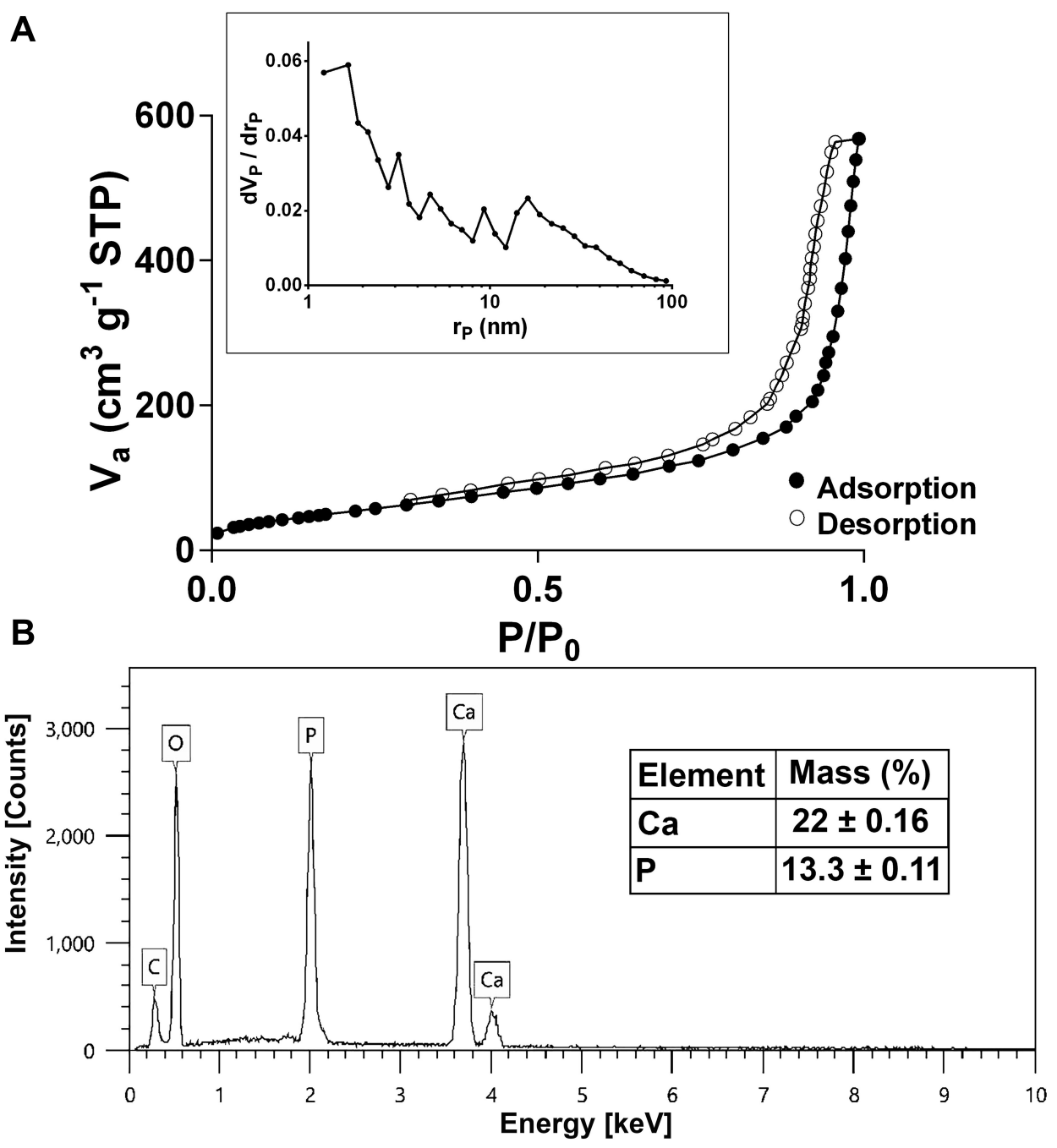

Figure I Characterization of HApN-F6 (A and B). Nitrogen-adsorption and -desorption isotherms for HApN-F6 (A), with inset showing pore-size distribution confirming the mesoporous profile. Energy-dispersive X-ray spectrum of HApN-F6 (B), showing typical peaks for hydroxyapatite elements.

factors indicates that the impact of one factor on a certain response is affected by the values of other factors.

\section{Influence on MPD}

Regression analysis for mean pore diameter (equation 3 in Table 3) showed aging temperature (C) to exert a positive effect $(p \leq 0.05)$ on MPD: this translated into an increase of MPD as aging temperature $(\mathrm{C})$ increased. A similar finding was reported by Mazinani et al, ${ }^{34}$ where raising the aging temperature of mesoporous silica to over $70^{\circ}-130^{\circ} \mathrm{C}$, resulted in larger pore diameters. This behavior can be related to the effect of temperature on interparticle connectivity and bridging (neck growth), with subsequent widening of pores as a result of possible dissolution and reprecipitation of nanoparticles. ${ }^{11}$ Regarding experimental responses in Table 2, an increase in MPD values mirrored the increase in aging temperature (C) only for longer aging ( $\mathrm{B}=24$ hours), but this pattern did not apply for $\mathrm{B}=1$ hour. This can be interpreted in light of interactions among different factors. The terms $1.65 \times \mathrm{BC}$ and $-0.43 \times \mathrm{ABC}$ showed significant $(p \leq 0.05)$ interactions, wherein the impact of BC on MPD exceeded all other variables. A possible explanation might be that pores widening due to interparticle neck growth and bridging is a time-influenced process. With less aging ( $\mathrm{B}=1$ hour), increasing temperature might have resulted in an increase in interparticle connectivity with no increase in pore diameter, which could have led to the overall decrease in MPD.

\section{Influence on $\mathrm{a}_{\mathrm{s}, \mathrm{BET}}$}

As identified from the negative terms of equation 4 in Table 3, all three independent variables exerted a negative impact on $\mathrm{a}_{\mathrm{s}, \mathrm{BET}}$ (all $p \leq 0.05$ ). As such, as values for any of 
Table 3 Regression Analysis Established in the Factorial Design for Optimization of Hydroxyapatite Nanoparticles Surface Area Attributes-

\begin{tabular}{|l|l|l|}
\hline Equation & Coded equations for each response ${ }^{\mathbf{a}, \mathbf{b}}$ & $\boldsymbol{R}^{\mathbf{2}}$ \\
\hline 3 & $\begin{array}{l}\text { MPD }(\mathrm{nm})=14.09-0.15 \times \mathrm{A}+0.16 \times \mathrm{B}+0.58 \\
\times \mathrm{C}+0.56 \times \mathrm{AB}+0.12 \times \mathrm{AC}+1.65 \times \mathrm{BC}- \\
0.43 \times \mathrm{ABC}\end{array}$ & 0.96 \\
\hline 4 & $\begin{array}{l}\mathrm{a}_{\mathrm{s}, \mathrm{BET}}\left(\mathrm{m}^{2} / \mathrm{g}\right)=166.31-13.28 \times \mathrm{A}-4.13 \times \mathrm{B}- \\
9.82 \times \mathrm{C}-4.72 \times \mathrm{AB}+12.26 \times \mathrm{AC}-1 \mathrm{I} .91 \times \\
\mathrm{BC}-10.17 \times \mathrm{ABC}\end{array}$ \\
\hline 5 & $\begin{array}{l}\mathrm{V}_{\mathrm{P}}\left(\mathrm{cm}^{3} / \mathrm{g}\right)=0.62-0.02 \times \mathrm{A}-0.07 \times \mathrm{B}-0.007 \\
\times C-0.009 \times \mathrm{AB}+0.04 \times \mathrm{AC}+0.01 \times \mathrm{BC}-\end{array}$ & 0.97 \\
& $0.05 \times \mathrm{ABC}$ & \\
\hline
\end{tabular}

Notes: ${ }^{a} A$, precursor concentration; $B$, aging time; $C$, aging temperature. ${ }^{b} p \leq 0.00$ I (overall model).

Abbreviations: MPD, mean pore diameter; $a_{\mathrm{s}, \mathrm{BET}}$, total specific surface area (Brunauer-Emmett-Teller); $V_{B}$ total pore volume.

the studied factors decreased, an increase in $\mathrm{a}_{\mathrm{s}, \mathrm{BET}}$ would ensue.

The negative effect of precursor concentration on surface area can be attributed to an increase in HApN size as a function of precursor concentration, ${ }^{35}$ with a consequent decrease in surface area. ${ }^{31}$ For $\mathrm{F} 3(\mathrm{~A}=0.06 \mathrm{M}, \mathrm{B}=1$ hour, and $\left.\mathrm{C}=50^{\circ} \mathrm{C}\right)$ and $\mathrm{F} 4(\mathrm{~A}=0.1 \mathrm{M}, \mathrm{B}=1$ hour, and $\mathrm{C}$ $=50^{\circ} \mathrm{C}$ ), increasing precursor concentration from $0.06 \mathrm{M}$ (F3) to $0.1 \mathrm{M}(\mathrm{F} 4)$ resulted in an increase in surface area (Table 2). This behavior can be interpreted in view of interactions among the independent variables under study: all interactions were statistically significant $(p \leq 0.05)$. The increase in surface area as precursor concentration increased at 1 -hour aging time, and $50^{\circ} \mathrm{C}$ aging temperature might have been an indirect result of particle collision, possible dispersion, and subsequent decrease in particle size. Interparticle collision, inevitable at high temperatures, might have been augmented at higher precursor concentrations, resulting in higher surface area of F4 than F3.

The inverse impact of aging time on surface area (equation 4 in Table 3) can be elaborated in terms of its effect on particle size. More specifically, the dissolutionreprecipitation expected during aging might have resulted in crystal ripening and inevitable increase in particle size, which might have reduced surface area. ${ }^{36}$ The same result was reported by Guo et al, where increasing aging time from 4 to 8 hours resulted in reduction of specific surface area of layered double-hydroxide crystals. ${ }^{37}$ Even though the hydroxides addressed in that study were chemically different from HApN, they were synthesized using precipitation from an aqueous solution under similar conditions to the HApN in our study. Also, the hydroxides prepared resembled HApN in their mesoporous nature and type IV nitrogen isotherm. The negative effect of aging time on surface area can only be seen for higher aging temperature $\left(\mathrm{C}=50^{\circ} \mathrm{C}\right)$. Increased aging time at ambient temperature $\left(\mathrm{C}=25^{\circ} \mathrm{C}\right)$ resulted in an increase in surface area. This might be ascribed to the combined effect of higher solubility of HApN crystals at lower temperature $\left(\mathrm{C}=25^{\circ} \mathrm{C}\right)^{38}$ and the hydrolysis and reprecipitation concomitant with longer aging time $\left(\mathrm{B}=24\right.$ hours). ${ }^{36}$ These processes occurring concurrently might have caused a reduction in particle size and hence larger surface area.

The negative effect of aging temperature on surface area can be interpreted via different hypotheses. One explanation is based on the direct impact of temperature on particle size, where increasing aging temperature results in higher surface energy of particles and subsequent particle coalescence. ${ }^{39}$ Another suggests that increasing temperature leads to the expulsion of trapped gases within HApN crystals, then subsequent particle densification would reduce surface area. ${ }^{11}$ The inverse impact of aging temperature on surface area was also reported by Kothapalli et $\mathrm{al}^{40}{ }^{40}$ where surface area of HApN crystals decreased as temperature increased from $25^{\circ} \mathrm{C}$ to $75^{\circ} \mathrm{C}$. Experimental values of responses (Table 2) show that increasing aging temperature from $25^{\circ} \mathrm{C}(\mathrm{F} 5)$ to $50^{\circ} \mathrm{C}$ (F4) exerted a positive effect on surface area at precursor concentration of $0.1 \mathrm{M}$ and aging time of 1 hour. This finding could be related to interactions among studied variables. More specifically, the AC interaction exerted a higher impact on $\mathrm{a}_{\mathrm{s}, \mathrm{BET}}$ than aging temperature alone. The higher surface area of F4 $\left(0.1 \mathrm{M}, 1\right.$ hour, and $\left.50^{\circ} \mathrm{C}\right)$ compared to $\mathrm{F} 5\left(0.1 \mathrm{M}, 1\right.$ hour, and $\left.25^{\circ} \mathrm{C}\right)$ could be explained by particles colliding at higher temperature $\left(50^{\circ} \mathrm{C}\right)$, associated with higher precursor concentration $(0.1 \mathrm{M})$, which might have resulted in possible particle redispersion and decrease in particle size. It is worth noting that the same behavior for F4 was detected for precursor concentration and could be interpreted in a similar manner. It should be mentioned that even with the simple preparation technique we employed, surface-area results obtained in our study exceeded those of other literature,${ }^{32,41}$ despite their more laborious preparation. This might have been due to the different precursors used in those studies and higher ranges of temperature applied. 


\section{Influence on $V_{P}$}

Regression analysis for $V_{P}$ (equation 5 in Table 3) showed both precursor concentration (A) and aging time (B) had negative effects $(p \leq 0.05)$ on $\mathrm{V}_{\mathrm{P}}$. An increase in either of these factors resulted in decreased $V_{P}$. The total pore volume calculated from the BET analysis of nitrogen-adsorption and -desorption isotherms reflects the amount of nitrogen adsorbed in the material in specific conditions. In the same context, the amount of adsorbed gas increases as the material surface area $\left(\mathrm{a}_{\mathrm{s}, \mathrm{BET}}\right)$ increases. Therefore, total pore volume can be related to surface area, and interpretation of the inverse impact of studied factors on HApN $a_{s, B E T}$ can be extrapolated to $V_{P}$. By examining responses for $V_{P}$ (equation 5 in Table 2), it can be pointed out that raising precursor concentration (A) resulted in a decrease in $\mathrm{V}_{\mathrm{P}}$, except for $\mathrm{F} 3$ $\left(0.06 \mathrm{M}, 1\right.$ hour, and $\left.50^{\circ} \mathrm{C}\right)$ and $\mathrm{F} 4\left(0.1 \mathrm{M}, 1\right.$ hour, and $50^{\circ}$ C). The same pattern was found for surface-area results of both formulations, and can be explained in similar fashion. In other words, the collision of particles inherent to high temperature coupled with high precursor concentrations might have resulted in a decrease in particle size and hence larger total pore volume for F4 compared to F3.

It is evident that surface-area parameters are greatly affected by the individual variables under study, namely precursor concentration, aging time, and aging temperature. Also, the conditions used in this study yielded mesoporous HAp particles with relatively higher surface area than literature-reported counterparts. ${ }^{32,41}$

\section{Scanning Electron Microscopy-Energy-Dispersive X- Ray Analysis}

The prepared HApN, HApN-F6, were analyzed using SEM-EDX and compared to standard HAp. The EDX spectrum (Figure 1B) exhibited the distinctive elemental peaks for $\mathrm{Ca}, \mathrm{O}$, and $\mathrm{P}$ with no other contaminating peaks, which established the purity of the prepared HAp. According to the percentage mass of calcium and phosphate, the Ca:P molar ratio (1.654) was in accordance with the established one (1.667) for HAp of natural bone. ${ }^{20}$

\section{Preparation and Optimization of DX/ HAp}

The interaction between HAp and DX, as well as other tetracyclines, takes place by drug adsorption on HAp as a result of van der Waals forces. ${ }^{14}$ In ethanol, the solvent used in our study, this process is reversible, with no effect on the chemical integrity of DX. ${ }^{14}$ DX/HAp were prepared by simple adsorption and optimized for drug loading by studying the effects of DX concentration and HApN surface-area properties.

\section{Effect of DX Concentration}

In order to optimize DX concentration for drug loading into HApN, we investigated three DX-loading concentrations: $1.33,3.33$, and $6.66 \mathrm{mg} / \mathrm{mL}$ in ethanol. For comparative purposes, HApN-F6 $\left(0.06 \mathrm{M}, 1\right.$ hour, and $\left.25^{\circ} \mathrm{C}\right)$ was selected for this study. Both drug loading and EE exhibited a significant $(p \leq 0.05)$ increase as DX-loading concentration was increased (Figure 2A). At $6.66 \mathrm{mg} / \mathrm{mL}$ DX concentration, EE reached its maximum value of $58.45 \% \pm 0.51 \%$ and maximum drug-loading value of $0.97 \pm 0.008 \mathrm{mg} / \mathrm{mg}$. Therefore, further drug-loading studies were conducted using $6.66 \mathrm{mg}$ / $\mathrm{mL}$ DX. The direct relation between DX concentration and adsorption behavior into HApN was in fact expected. Drugs interaction with HAp is a complicated process that depends highly on the equilibrium concentration of the drug, among other factors. ${ }^{26}$ It has been hypothesized that increasing the drug concentration enhances the adsorption between the drug and HApN surface as adsorbent. ${ }^{42}$

Langmuir and Freundlich adsorption models were used for data fitting. Theoretically, the Langmuir model describes adsorption that takes place as a monolayer coverage on finite homogeneous adsorption sites of equal energy, with no interaction between adsorbate molecules. ${ }^{25}$ On the other hand, the Freundlich model describes an indefinite adsorption pattern taking place in multiple layers on a heterogeneous surface. ${ }^{25}$ Results (Table 4) revealed the Langmuir isotherm model fitted our experimental data with a slightly higher $R^{2}$ value than the Freundlich model. This implies that DX adsorption into HApN could possibly follow a monolayer pattern. Similarly, Li et al reported that adsorption of tetracycline into iron-incorporated HAp followed a Langmuir pattern. ${ }^{43}$ However, the closeness of $R^{2}$ values between the models indicates that this parameter might not be uniquely indicative of model verification. ${ }^{25}$

\section{Effect of Surface-Area Parameters}

The specific surface area of HApN greatly impacts their adsorptive potential and hence drug-loading capacity. We examined the dependence of drug content for DX/HAp on different surface-area parameters, where all eight formulations prepared for the factorial design (Table 2) were used for drug loading at $6.66 \mathrm{mg} / \mathrm{mL}$ DX concentration and DX to HApN ratio of 2:1. Afterward, we conducted correlation and linear regression analyses to investigate linear associations between each total specific surface area and total pore 


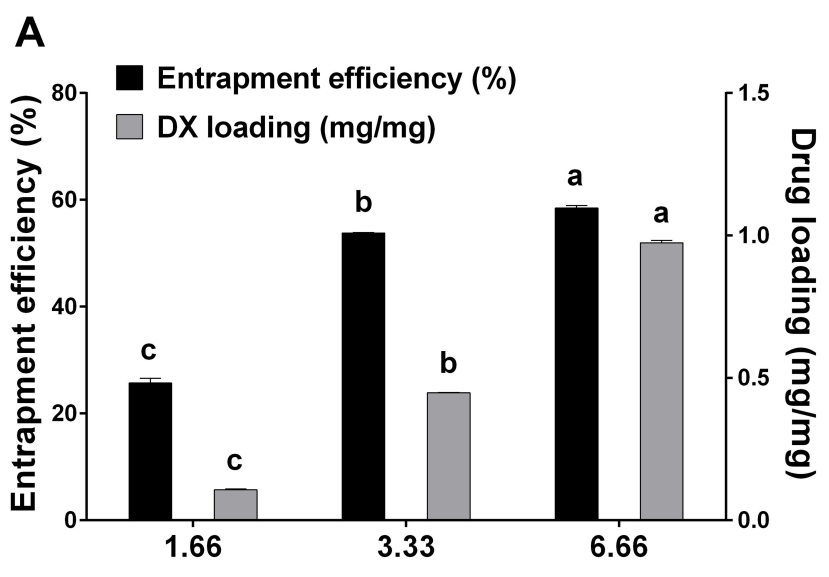

Concentration of DX solution $(\mathrm{mg} / \mathrm{ml})$
B

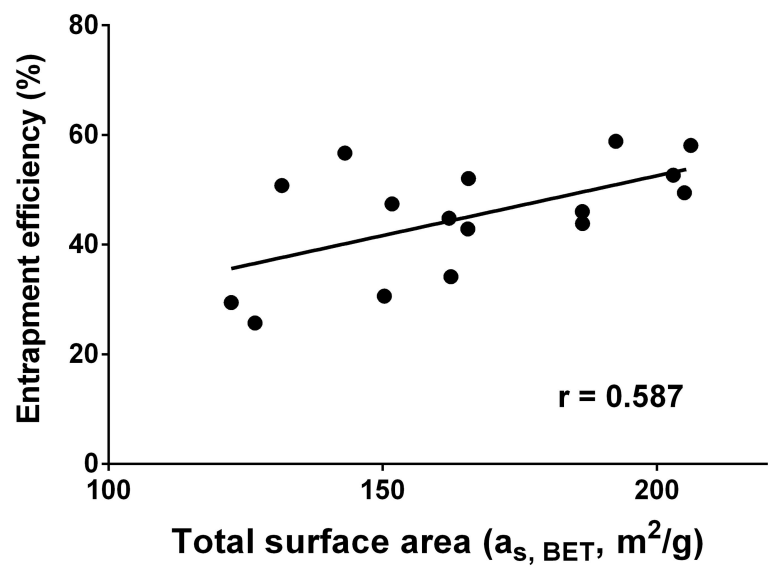

Figure 2 Optimization of doxycycline (DX) loading on hydroxyapatite nanoparticles (A and B). Drug content of DX in HApN-F6 (A), expressed as drug amount per unit weight of loading hydroxyapatite nanoparticles, showing a direct relationship to drug concentration. $n=3$, $p \leq 0.05$, bars in same series bearing different letters were significantly different: $a>b>c$. Linear regression plot for surface area of the eight formulations of hydroxyapatite nanoparticles against DX-entrapment efficiency (B), confirming a direct positive correlation (two sets of measurements).

volume for blank HApN formulations (Table 2) on DX drug loading and EE for the corresponding DX/HAp prepared. Results indicated that Pearson's correlation coefficient, reflecting the intensity of linear association, exceeded 0.5 . This implied the presence of a positive correlation among the studied variables, all correlations were significant $(p \leq 0.05)$. In other words, drug loading and EE of DX into HApN increased in a linear pattern as each total specific surface area $\left(\mathrm{m}^{2} / \mathrm{g}\right)$ or total pore volume $\left(\mathrm{cm}^{3} / \mathrm{g}\right)$ increased (representative plot in Figure 2B). Indeed, the F6 HApN formulation showed the highest DX loading $(0.97 \pm 0.008 \mathrm{mg} / \mathrm{mg})$ and $\mathrm{EE}(58.45 \% \pm 0.51 \%)$. The same formulation (F6 in Table 2) resulted in the highest total pore volume $\left(0.84 \pm 0.01 \mathrm{~cm}^{3} /\right.$ g) and the second-highest total specific surface area (199.34 $\pm 9.66 \mathrm{~m}^{2} / \mathrm{g}$ ), with a slight difference from $\mathrm{F} 8$, with highest specific surface area of $204 \pm 1.44 \mathrm{~m}^{2} / \mathrm{g}$. Similar results were reported by Tabassum et al, ${ }^{33}$ where ibuprofen loading was maximum for HAp particles with higher surface area and porosity.

Table 4 Adsorption-Isotherm Parameters for Doxycycline Adsorption in Hydroxyapatite Nanoparticles (F6)

\begin{tabular}{|l|l|l|l|l|l|}
\hline \multicolumn{2}{|l|}{ Langmuir model $^{\mathrm{a}}$} & \multicolumn{3}{l|}{ Freundlich model $^{\mathrm{b}}$} \\
\hline$R^{2}$ & $\begin{array}{l}\mathbf{Q}_{\max } \\
(\mathbf{m g} / \mathrm{mg})\end{array}$ & $\begin{array}{l}\mathrm{K}_{\text {ads }} \\
(\mathbf{m L} / \\
\mathbf{m g})\end{array}$ & $R^{2}$ & $\begin{array}{l}\mathbf{K}_{\mathrm{F}}(\mathbf{m g} / \mathbf{m g}) \times \\
\left.(\mathbf{m g} / \mathbf{m L})^{\mathbf{n}}\right)\end{array}$ & $\mathbf{n}$ \\
\hline 0.995 & 93.44 & 0.001 & 0.986 & 0.08 & 0.77 \\
\hline
\end{tabular}

Notes: ${ }^{a} Q_{\max }$, the maximum amount of adsorbate per unit weight of adsorbent at equilibrium; $\mathrm{K}_{\mathrm{ads}}$, Langmuir constant (related to the adsorption rate). ${ }^{\mathrm{b}} \mathrm{K}_{\mathrm{F}}$ Freundlich constant (indicates the adsorption capacity of the adsorbent).
To sum up, DX loading on HApN was indeed influenced by drug-loading concentration and surface-area properties of HApN as carrier. The interplay of both factors, among others, would help optimize the use of HApN as a drug-delivery device. In this study, DX-loaded F6 (DX/HAp-F6) was selected as the optimum formulation and used for further experiments.

\section{Preparation and Optimization of Composite DX/HAp/PCL}

The use of emulsification has been commonly applied to prepare polymeric nanoparticles. ${ }^{23,44}$ Composite DX/HAp/ PCL particles in this study were prepared using $\mathrm{S} / \mathrm{O} / \mathrm{W}$ emulsion-solvent evaporation, where DX was introduced into the system in a dispersed form. We prepared four formulations (Table 5) to investigate the effect of PCL concentration in the organic phase and the presence of $4 \% \mathrm{NaCl}$ in the aqueous phase on DX EE. It is worth noting that we preliminarily investigated higher $\mathrm{NaCl}$ concentrations (data not shown). However, higher $\mathrm{NaCl}$ concentrations resulted in precipitation of either $\mathrm{NaCl}$ or PVA from solution and were thus excluded from further trials. Even though $\mathrm{W}_{1} / \mathrm{O}$ / $\mathrm{W}_{2}$ is one of the most popular techniques applied for loading hydrophilic drugs into hydrophobic polymers, this method still suffers poor drug EE. To gain more insight, DX-loaded PCL particles were prepared using $\mathrm{W}_{1} / \mathrm{O} / \mathrm{W}_{2}$ (DX/PCL) applying two PCL concentrations (Table 5). Results of two-way ANOVA for EE data of composite DX/HAp/PCL showed that the studied factors, ie, PCL concentration in the organic phase and the presence of $4 \% \mathrm{NaCl}$ in the aqueous 
phase, exerted significant $(p \leq 0.001)$ negative and positive effects, respectively, on EE. Indeed, EE for composite DX/ HAp/PCL particles (Table 5) ranged from $69.42 \% \pm 2.68 \%$ to $94.77 \% \pm 1.23 \%$, with the highest value observed for DX/ HAp/PCL-A prepared using $1 \%$ PCL and $4 \% \mathrm{NaCl}$.

The inverse relationshiop between PCL concentration and DX EE has previously been reported. ${ }^{45}$ It can be attributed to the easy penetration of water through the amorphous region of the semicrystalline polymer PCL, more obvious at higher concentrations, during solvent evaporation, and consequent DX diffusion into the aqueous external phase. ${ }^{46}$ However, Raval et al ${ }^{47}$ reported an increase in DX EE as PCL concentration increased. This discrepancy might have been due to the higher PCL concentration range $(2 \%-20 \%)$ applied by Raval et al than used in this work $(1 \%-2 \%)$. In the same context, the increase in DX EE as a result of adding $4 \% \mathrm{NaCl}$ might have been due to two related phenomena. First, the common ion equilibrium of chloride could have affected the solubility of DX hydrochloride salt in the external aqueous phase. ${ }^{46}$ Moreover, adding an electrolyte might have rendered the external aqueous phase more hypertonic, resulting in less DX diffusion outwards and consequently confining DX in the polymeric matrix. A similar finding has previously been reported, ${ }^{45}$ where adding $4 \% \mathrm{NaCl}$ to the external aqueous phase increased DX EE in PCL/ PLGA nanoparticles from $47 \%$ to $70 \%$. As can be seen in Table 5, EE of DX/PCL prepared by $\mathrm{W}_{1} / \mathrm{O} / \mathrm{W}_{2}$ were significantly $(p \leq 0.05)$ lower than other composite particles of $\mathrm{DX} / \mathrm{HAp} / \mathrm{PCL}$. Also, it is worth noting that increasing PCL concentration had no effect on DX EE. In the $\mathrm{W}_{1} / \mathrm{O} /$ $\mathrm{W}_{2}$ technique, a hydrophilic drug in aqueous solution is

Table 5 Entrapment Efficiency of Doxycycline for Polycaprolactone Particles Under Different Study Conditions

\begin{tabular}{|c|c|c|c|}
\hline \multirow{2}{*}{$\begin{array}{l}\text { Formulation } \\
\text { code }\end{array}$} & \multicolumn{2}{|c|}{ Formulation variables } & \multirow{2}{*}{$\begin{array}{l}\text { Entrapment } \\
\text { efficiency* } \\
\text { (\%) }\end{array}$} \\
\hline & $\begin{array}{l}\text { PCL } \\
\text { concentration in } \\
\text { organic phase (\%) }\end{array}$ & $\begin{array}{l}4 \% \\
\mathrm{NaCl}\end{array}$ & \\
\hline DX/HAp/PCL-A & I & + & $94.77 \pm 1.23^{\mathrm{a}}$ \\
\hline DX/HAp/PCL-B & I & - & $84.07 \pm 0.53^{\mathrm{b}}$ \\
\hline DX/HAp/PCL-C & 2 & + & $80.93 \pm 0.82^{b}$ \\
\hline DX/HAp/PCL-D & 2 & - & $69.42 \pm 2.68^{c}$ \\
\hline DX/PCL-A & I & + & $7.20 \pm 0.55^{\mathrm{d}}$ \\
\hline DX/PCL-B & 2 & + & $7.83 \pm 0.99^{d}$ \\
\hline
\end{tabular}

Notes: $*_{n}=3 ; p \leq 0.05$; values bearing different letters were significantly different: a > $b>c>d$.

Abbreviations: PCL, polycaprolactone; DX, doxycycline; HAp, hydroxyapatite. emulsified in the polymer organic phase, then further emulsified in an external aqueous phase. ${ }^{46}$ As such, it is postulated that poor DX EE is a result of drug in solution diffusing to the external aqueous phase during organic solvent evaporation.

The use of HApN in this work enabled the loading of DX into PCL as composite DX/HAp/PCL using the S/O/ $\mathrm{W}$ technique via the prior anchoring of $\mathrm{DX}$ to HApN by adsorption. This made possible the entrapment of DX into PCL particles with the highest EE reported to date in the literature.

\section{In Vitro Drug Release}

Various composite systems have been implemented as drugdelivery devices for bone-regeneration therapy. For all these systems, it is crucial that they are able to provide controlled release of the loaded drug into the local site of action. For DX specifically, high doses exert cytotoxic actions and negative effects on cell differentiation in vitro. ${ }^{31}$ Therefore, it was essential that we achieve controlled release of DX, in order to ameliorate its dose-dependent toxicity while augmenting its osteogenic actions. In order to investigate the effect of PCL concentration on DX release, in vitro release experiments were run for each of the composites DX/HAp/ PCL-A and DX/HAp/PCL-B, with PCL concentrations 1\% and $2 \%$, respectively, and compared these to DX/HAp-F6. It should be noted that the poor EE of DX into DX/PCL impeded the investigation of DX release from these formulations. As shown in Figure 3, the release profile of DX from DX/HAp-F6 was biphasic in nature, where the initial release of $11.14 \% \pm 0.63 \%$ after 2 hours progressed in a relatively controlled manner, reaching $22.65 \% \pm 6.15 \%$ after 10 days. That was then followed by a higher-rate release pattern, reaching $91 \% \pm 3.20 \%$ after 24 days. The reason for the initial relatively controlled DX release from DX/HAp-F6 might have been the high affinity of DX with the HApN surface and DX adsorption within HApN mesopores. ${ }^{2}$ However, as the adsorbed DX is released into the medium, more hosting HApN mesopores are exposed, with a consequent increase in water flowing through the porous system. This might have resulted in the high DX-release rate in the second phase.

The release of DX from both composites DX/HAp/ PCL-A and DX/HAp/PCL-B (Figure 3) also followed a biphasic pattern that comprised an initial burst release phase of DX followed by a prolonged controlled-release phase. The burst-release phase for DX/HAp/PCL-A and DX/HAp/PCL-B involved the rapid release of $39.13 \%$ 
$\pm 0.76 \%$ and $34.95 \% \pm 0.4 \% \mathrm{DX}$, respectively, during the first 2 hours with DX/HAp/PCL-A significantly $(p \leq 0.05)$, higher than DX/HAp/PCL-B. This was followed by longer controlled release of DX spanning 24 days, during which $73.89 \% \pm 7.86 \%$ and $62.00 \% \pm 7.23 \%$ DX was cumulatively released from DX/HAp/PCL-A and DX/HAp/PCL-B, respectively. No significant $(p \leq 0.05)$ difference was further detected between these release patterns. The initial burst release of DX from composite PCL particles could have been a result of the semicrystalline nature of PCL, allowing water penetration through the polymer amorphous region and the subsequent diffusion of DX near the surface of the particles. ${ }^{46}$ The controlled release afterward was possibly due to the slow diffusion of DX from within the hydrophobic polymeric matrix into the release medium. It is worth mentioning that DX release from both composite formulations exhibited less burst release, longer overall duration, and more DX retention and controlled release than other DX-loaded PCL particles. ${ }^{45-47}$ This could be associated with the preparation technique applied in our study, $\mathrm{S} / \mathrm{O} / \mathrm{W}$, that might have offered more confinement of DX within the polymeric matrix than the $\mathrm{W}_{1}$ / $\mathrm{O} / \mathrm{W}_{2}$ technique used in other reports.

Even though the initial DX release from DX/HAp-F6 was relatively slow, its incorporation into PCL resulted in a significant $(p \leq 0.05)$ increase in DX burst release. This might be ascribed to the sonication applied during polymeric particle preparation, which could have resulted in the presence of more DX near the polymeric surface. However, the incorporation of DX/HAp-F6 into the

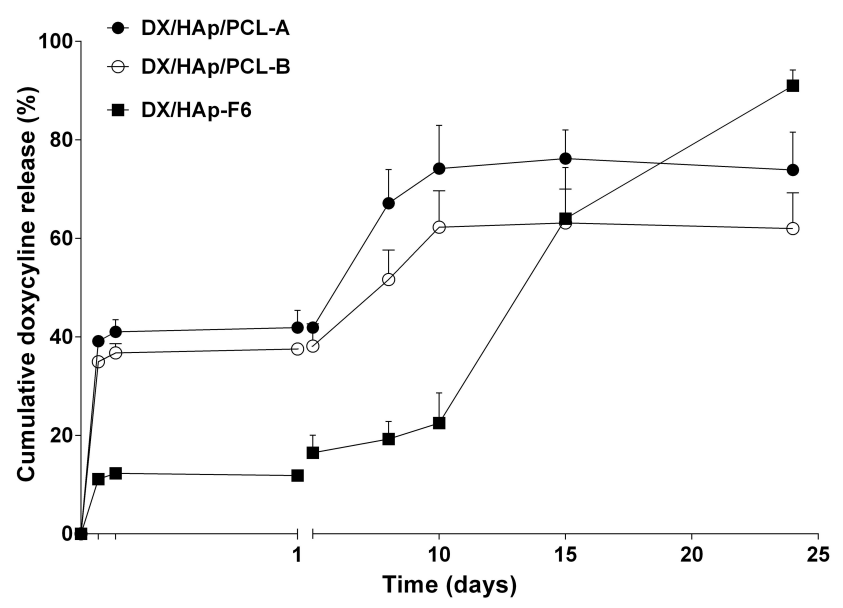

Figure 3 In vitro cumulative release profile of doxycycline from DX/HAP/PCL-A, DX/HAp/PCL-B and DX/HAp-F6, showing initial burst release followed by prolonged controlled release of $\mathrm{DX}(n=3)$. hydrophobic matrix provided more control of DX-release rate, with consequently lower extent of release over the whole profile duration. Both composite formulations expressed generally similar release profiles, and hence composite DX/HAp/PCL-A, having been prepared using lower PCL concentration, was selected for further studies.

\section{Characterization of Selected}

\section{Formulations}

\section{X-Ray Diffraction}

The crystalline state of $\mathrm{HApN}$ is an important factor affecting its behavior as a bioactive material. Crystalline $\mathrm{HApN}$ have been reported to offer a more efficient substrate for BMSCs to adhere, proliferate, and differentiate into osteogenic lineage than amorphous calcium phosphate. $^{48}$ XRD analysis for HApN-F6, DX/HAp-F6, and composite DX/HAp/PCL-A was carried out (Figure 4). Diffraction patterns for HApN-F6 showed accordance with the Crystallography Open Database (COD) reference HAp (96-900-2215): the specific peaks of (002), (211), (202), and (310) reflections were distinguished at $2 \theta=25.9^{\circ}, 31.79^{\circ}, 34.11^{\circ}$, and $39.83^{\circ}$, respectively. This indicates that the HApN-F6 prepared using wet precipitation was of a highly pure nature, with no impurities or secondary calcium phases. Furthermore, the presence of broad peaks compared to the reference pattern confirmed the nanoscale of the HAp prepared using chemical precipitation. $^{39}$

Regarding DX/HAp-F6 and composite DX/HAp/PCLA, XRD patterns also featured peaks for HAp implying that HApN crystallinity has been retained in both formulations. In addition, the presence of a diffraction peak at $2 \theta=23^{\circ}$, characteristic of $\mathrm{DX},{ }^{49}$ reflected the presence of drug in its crystalline form in both formulations - DX/ HAp-F6 and composite DX/HAp/PCL-A. On the basis that a crystalline state offers more drug stability than amorphous ones, ${ }^{50,51}$ our results proved beneficial. Further, the presence of drug in a crystalline form provides localized drug depots for controlling drug release, ${ }^{51}$ which could further explain the controlled in vitro DX-release pattern and greatly served our research objective. The fact that DX retained its crystalline structure in both formulations could possibly be due to DX interaction with HApN. As previously mentioned, adsorption of DX from its ethanolic solutions on HApN takes place via van der Waals forces without the involvement of any significant ionic interaction. ${ }^{14}$ This might be the reason that no change in 


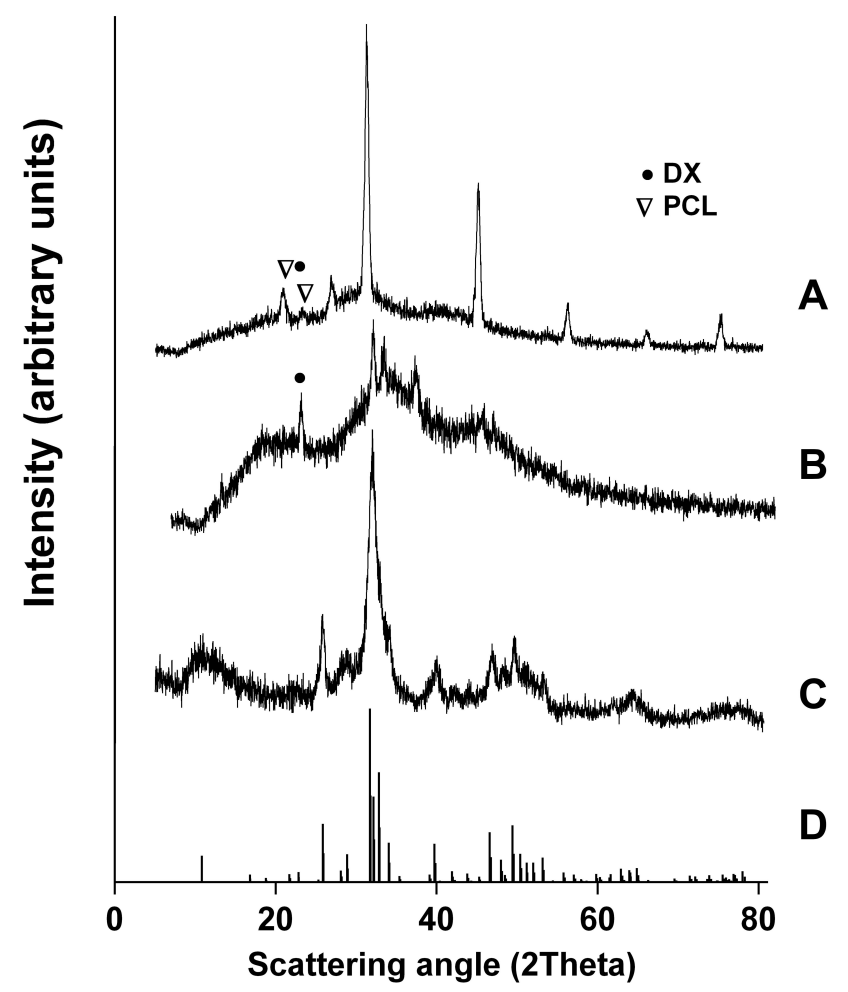

Figure 4 X-ray diffraction patterns for composite DX/HAp/PCL-A (A), DX/HApF6 (B), HApN-F6 (C), and COD reference hydroxyapatite (96-900-2215) (D).

crystal behavior was detected in DX/HAp-F6 or composite DX/HAp/PCL-A, where DX already adsorbed on HApN was used for further loading into PCL. This hypothesis can be confirmed by similar results previously reported, ${ }^{52}$ where XRD patterns for both cholecalciferol-loaded HAp and cholecalciferol-HAp-poly(lactic-co-glycolic acid) (PLGA) nanoparticles exhibited characteristic peaks for the loaded cholecalciferol. Interestingly, drug loading in that study was implemented by simple physical precipitation of cholecalciferol from acetone solution on HAp, then further embedding into PLGA without significant chemical interaction, in a similar manner to the technique adopted in our study. The same justification can be extrapolated to the opposite results obtained by Raval et al, ${ }^{47}$ where XRD patterns of DX-loaded PCL microspheres presented no DX-specific peaks as a result of their amorphization. Particles in Raval et al's study were prepared using double emulsion involving the use of an aqueous DX solution and its subsequent dispersion in amorphous state.

It can be seen in Figure 4 that DX/HAp-F6 diffractometry presents a prominent hump, which might have resulted from XRD broadening of the amorphous sample substrate. ${ }^{53}$ Diffractometry for composite DX/HAp/PCL-A reflected additional peaks at $2 \theta=21.3^{\circ}$ and $23.9^{\circ}$, which are associated with scattering of the PCL crystalline region. ${ }^{54}$ Also, given the fact that PCL is a semicrystalline polymer, the slight hump in the XRD pattern shown can be attributed to the amorphous phase of PCL, as well as the nanoscale of the prepared particles, as previously reported. $^{54}$

Overall, the prepared formulations indeed retained the crystalline structure for their organic (DX and PCL) and inorganic (HApN) components. Also, the absence of impurities implies that the preparation technique resulted in minimum crystalline changes in the prepared particles.

\section{Transmission Electron Microscopy}

In order to evaluate the morphology and particle-size distribution for HApN-F6, DX/HAp-F6, and composite DX/HAp/ PCL-A, TEM was performed, as the most accurate method for size determination. ${ }^{55}$ As seen in Figure $5 \mathrm{~A}-\mathrm{C}$, both HApN-F6 and DX/HAp-F6 can be described as rodlike particles, while composite DX/HAp/PCL-A particles exhibited spherical morphology. Particle-size distribution for all formulations (Figure 5D-F) demonstrated a relatively unimodal pattern, all in the nanoscale range. The average length and width for DX/HAp-F6 were $111.74 \pm 21.88 \mathrm{~nm}$ and 38.56 $\pm 6.42 \mathrm{~nm}$, respectively, significantly larger $(p \leq 0.001)$ than corresponding values for HApN (length $90.12 \pm 15.22 \mathrm{~nm}$, width $30.24 \pm 5.97 \mathrm{~nm}$ ). This was expected, due to DX loading by adsorption onto HApN surfaces. The average diameter for the composite DX/HAp/PCL-A was $99.79 \pm 20.36 \mathrm{~nm}$. In previous reports, ${ }^{56}$ it has been demonstrated that nanosized particles can afford better structural bioactivity and hence osteoconductivity. They provide more efficient cellular attachment, proliferation, and osteogenic differentiation. On this basis, the nanoscale of the particles in this study is expected to favor osteogenic application.

\section{Fourier-Transform Infrared Spectroscopy}

FTIR analysis was conducted for HApN-F6, DX/HApF6, and composite DX/HAp/PCL-A. For comparative purposes, pristine DX, PCL, and physical mixtures of DX and HApN-F6 (PM-A), DX, HApN-F6, and PCL (PM-B) were analyzed (Figure 6). The spectrum for HApN-F6 showed bands for the stretching of hydroxyl groups at $653 \mathrm{~cm}^{-1}$ and $3,578 \mathrm{~cm}^{-1}$. The broad bands at $1,000-1,100 \mathrm{~cm}^{-1}$ and $3,250-3,500 \mathrm{~cm}^{-1}$ correspond to phosphate stretching and adsorbed water, ${ }^{57}$ respectively. Also, $\mathrm{C}=\mathrm{O}$ group stretching was identified at $872 \mathrm{~cm}^{-1}$, $1,418 \mathrm{~cm}^{-1}$, and $1,457 \mathrm{~cm}^{-1}$. The $\mathrm{CO}_{3}{ }^{2-}$ group 

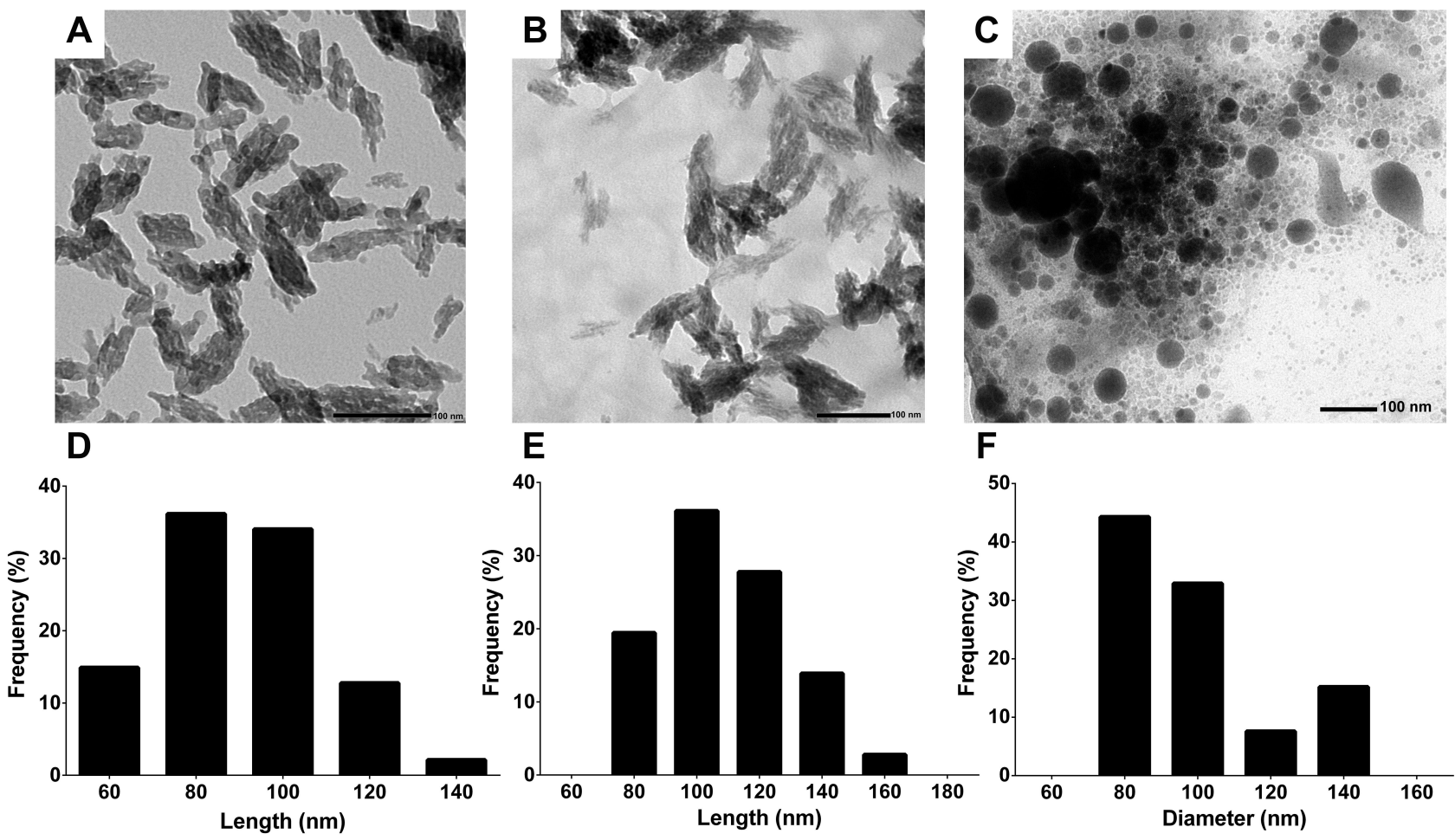

Figure 5 Particle-size analysis for the prepared formulations (A-F) confirming the nanosize range. Transmission electron microscopy images for HApN-F6 (A), DX/HAp-F6 (B), and composite DX/HAp/PCL-A (C). Histograms of size measurements for HApN-F6 (D), DX/HAp-F6 (E), and composite DX/HAp/PCL-A (F): $n=50$ particles.

bands could have been the result of contamination from atmospheric $\mathrm{CO}_{2}$ or the organic sample carrier ${ }^{58}$ and not inherent of the prepared particles, which was evident from SEM-EDX results. For the pristine DX spectrum, characteristic bands for $\mathrm{C}-\mathrm{H}$ stretching are shown at $2,881 \mathrm{~cm}^{-1}$ and $2,917 \mathrm{~cm}^{-1}$, while $\mathrm{O}-\mathrm{H} / \mathrm{N}-\mathrm{H}$ stretching is presented at $3,305 \mathrm{~cm}^{-1}$. Bands corresponding to aromatic $\mathrm{N}-\mathrm{H}$ bending and primary amide $\mathrm{N}-\mathrm{H}$ bending were found at $1,576 \mathrm{~cm}^{-1}$ and $1,669 \mathrm{~cm}^{-1}$, respectively. Also, bands corresponding to $\mathrm{C}=\mathrm{O}$ stretching, $-\mathrm{CH}_{2}$ bending, and $\mathrm{C}-\mathrm{H}$ bending were seen at $1,609 \mathrm{~cm}^{-1}$, $1,457 \mathrm{~cm}^{-1}$, and $1,329 \mathrm{~cm}^{-1}$, respectively. Finally, the bands at $1,174 \mathrm{~cm}^{-1}$ and $1,217 \mathrm{~cm}^{-1}$ corresponded to $\mathrm{C}-$ $\mathrm{N}$ stretching. ${ }^{46}$ The PCL spectrum showed stretching of the $\mathrm{C}-\mathrm{O}-\mathrm{C}$ group at $1,163 \mathrm{~cm}^{-1}$, the $\mathrm{C}=\mathrm{O}$ group at $1,724 \mathrm{~cm}^{-1}$, and the $\mathrm{C}-\mathrm{H}$ group at both $2,869 \mathrm{~cm}^{-1}$ and $2,945 \mathrm{~cm}^{-1.59}$ As can be seen in Figure 6, spectra for DX/HAp-F6 and composite DX/HAp/PCL-A showed all peaks for their individual components. Notably, the peaks for HAp hydroxyl groups and DX amine groups presented some slight shifting, which implies possible hydrogen-bond formation. In comparison, the corresponding physical mixtures PM-A and PM-B showed combinations of all ingredients; however, DX peaks demonstrated the highest intensity, probably due to the processing technique.

These findings generally confirm the formation of HAp. Also, it was established that DX and HApN maintained their chemical stability in both DX/HAp-F6 and the composite DX/HAp/PCL-A.

\section{Determination of Hydroxyapatite Content}

It was important to determine the HAp-reaction yield of HApN-F6, prepared using wet chemical precipitation. In addition, DX/HAp-F6 and composite DX/HAp/ PCL-A were analyzed for quantification of HAp content. The reaction yield of HApN-F6 was determined to be $80.31 \% \pm 10.81 \%$, which confirmed the wet precipitation adopted to be a reliable preparation technique with high process yield. Furthermore, incorporation of HApN into DX/HAp-F6 and composite DX/HAp/PCLA was determined to be $81.65 \% \pm 2.81 \%$ and $60.26 \%$ $\pm 8.02 \%$, respectively. It should be noted that the relatively lower incorporation for composite DX/HAp/ PCL-A compared to DX/HAp-F6 could have be due to to the steps conducted during composite preparation and probable concomitant loss. 


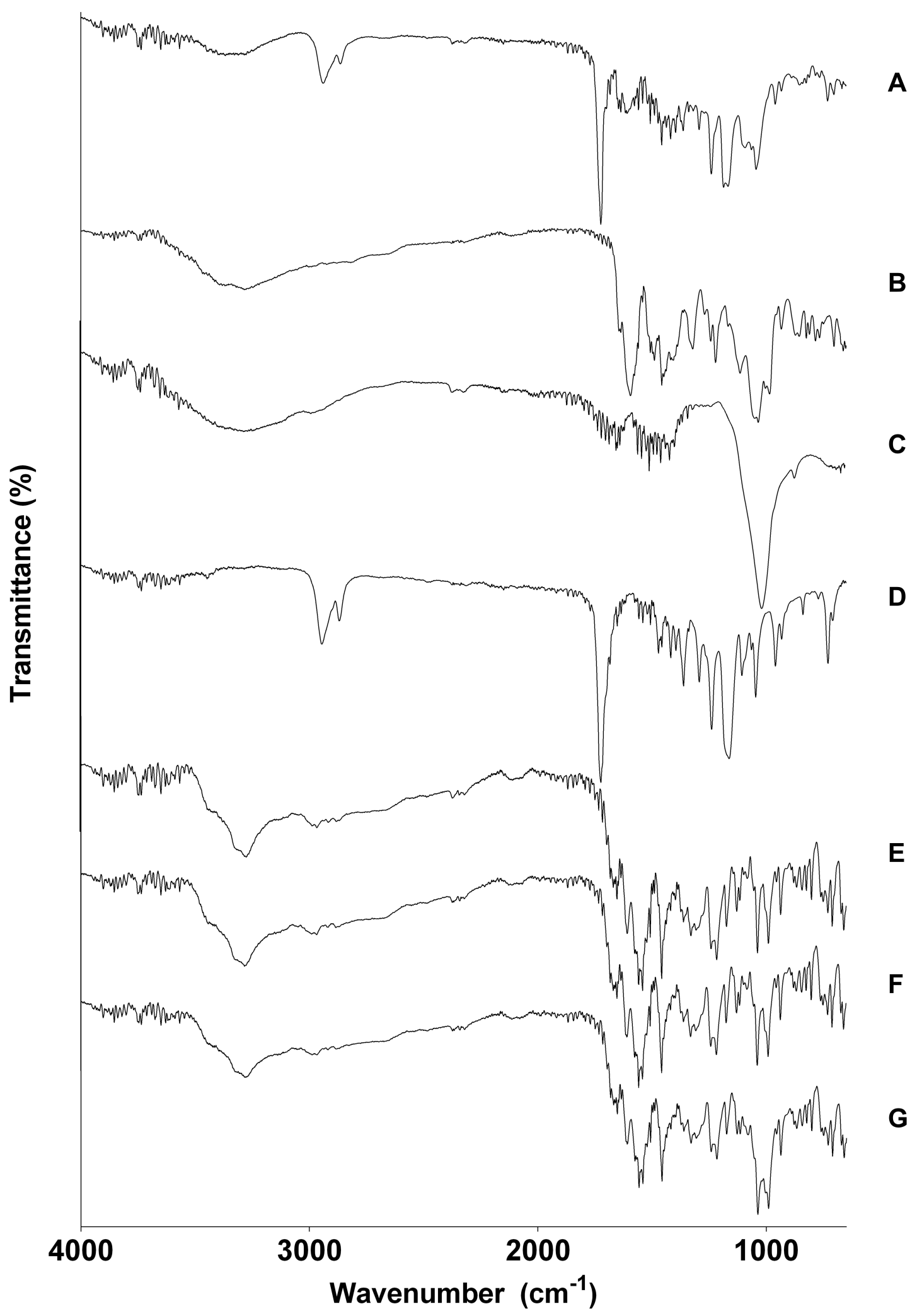

Figure 6 Fourier-transform infrared spectra for composite DX/HAp/PCL-A (A), DX/HAp-F6 (B), HApN-F6 (C), PCL (D), DX (E), PM-B - DX + HApN-F6 + PCL (F), and PM-A - DX + HAPN-F6 (G). 


\section{In Vitro Cell Culture}

Mesenchymal stem cells obtained from BM represent an efficient source of osteoprogenitor cells. ${ }^{12}$ Studying the differentiation of these cells in the presence of various biomaterials can be informative regarding the prospective osteogenicity of those materials. ${ }^{60}$ Generally, the transformation of osteoprogenitors into osteoblasts is a dynamic process that takes place over three main stages: proliferation, matrix maturation, and matrix mineralization. ${ }^{12}$ In this work, BMSCs previously isolated and immunologically characterized for mesenchymal multipotency by our team ${ }^{19}$ were cultured then propagated into a homogeneous cell population. Osteogenic lineage was then induced using ODM. BMSCs were used to assess osteogenic potential for treatment groups - DX solution, DX/HApF6, and composite DX/HAp/PCL-A - compared to appropriate control groups. To this end, various osteogenesis stages were characterized through different experiments.

\section{Cell Viability and Proliferation}

During the first stage of osteogenesis, osteoprogenitor cells undergo proliferation and collagen synthesis for subsequent ECM maturation. ${ }^{12}$ The MTT assay is an efficient technique for assessing cell metabolic activity. It quantifies cell viability with formazan as product to determine the mitochondrial dehydrogenase activity of viable cells. ${ }^{9}$ In this work, we used MTT assays to assess the influence of the applied formulations on the viability and proliferation of cells. ${ }^{9}$ Although cell viability was determined after 48 hours, this study could be used as a rough estimate of further cell behavior over longer durations. Results (Figure 7A) revealed that DX solution at $100 \mu \mathrm{g} / \mathrm{mL}$ exerted a cytotoxic effect on BMSCs, as evident from the significant $(p \leq 0.05)$ decrease in cell viability compared to the control group (CCM). The dose-dependent cytotoxic effect of DX has been previously reported, ${ }^{9}$ where DX solution at $25 \mu \mathrm{g} / \mathrm{mL}$ delayed the proliferation of human BM cells. Another study ${ }^{10}$ demonstrated that treatment with $100 \mu \mathrm{M}$ DX solution (corresponding to $48 \mu \mathrm{g} / \mathrm{mL}$ ) resulted in a very limited number of murine osteoprecursor cells. Notably, the discrepancy among DX toxic concentrations can be related to the difference in cell systems used (human or animal cells), overall study duration, and consequent variation in cellular permeability and sensitivity. Although not yet established, cytotoxic DX effect might be due to its ability to inhibit mitochondrial biogenesis in mammalian cells. ${ }^{61}$ Interestingly, DX solution at 50 $\mu \mathrm{g} / \mathrm{mL}$ (Figure 7A) presented a significant $(p \leq 0.05)$ increase in cell proliferation. The reason might be the modifications employed by DX on the collagenous ECM as a result of matrix-metalloproteinase inhibition, ${ }^{18}$ which probably outweighed its cytotoxic effect manifested at higher concentrations.

As demonstrated in Figure 7A, DX/HAp-F6 showed proliferative action on BMSCs at $50 \mu \mathrm{g} / \mathrm{mL} \mathrm{DX}$, resulting in cell viability that was significantly $(p \leq 0.05)$ higher than DX solution at the same concentration. This can be attributed to the proliferative action exerted by HApN as biomaterials, probably as a result of adsorption of serum proteins found in culture medium providing high local concentrations of these proteins, with subsequent enhancement of cell growth. ${ }^{62}$ On the other hand, it could be that the cytotoxic effect of $100 \mu \mathrm{g} / \mathrm{mL}$ DX loaded in DX/HAp-F6 was ameliorated both by the counteractive proliferative action of HAp and the incorporation of DX within the delivery system, which modified its release pattern and limited its direct contact with cells. In addition, composite DX/HAp/PCL-A at $100 \mu \mathrm{g} / \mathrm{mL}$ DX afforded significantly $(p \leq 0.05)$ higher cell viability, probably due to PCL proliferative action, ${ }^{63}$ more pronounced at higher PCL amounts in particles corresponding to $100 \mu \mathrm{g} / \mathrm{mL}$ DX. Nanofibers of plain PCL have been reported to improve the proliferation of hosted stem cells. ${ }^{63}$ Therefore, it is possible that PCL-based nanosystems can act as an anchor to provide more cell interaction and support cell proliferation. ${ }^{64}$ Moreover, the further incorporation of DX/HAp-F6 within the polymeric matrix in composite DX/HAp/PCL-A might have resulted in less contact between DX and BMSCs, hence ameliorating cytotoxic DX effects at this high concentration. Given these results, $50 \mu \mathrm{g} / \mathrm{mL}$ DX concentration was selected for all formulations and was further used in other experiments.

\section{ALP-Activity Assays}

As osteogenesis proceeds, synthesis of the ECM takes place in the second stage with the intense production of bone-ECM proteins, including ALP. ALP is an early osteoblast-differentiation marker contributing to initiation of mineralization ${ }^{12}$ by providing large amounts of phosphate ions required for the onset of mineral deposition. ${ }^{18}$ As identified in Figure 7B, ALP activity for all groups after culture for 10 days significantly $(p \leq 0.05)$ exceeded activity after 5 days, which conforms with ALP general behavior in stem cells undergoing differentiation toward osteoblasts. ${ }^{18}$ Notably, ALP activity in the control (CCM) group exhibited an increase over culture time that 

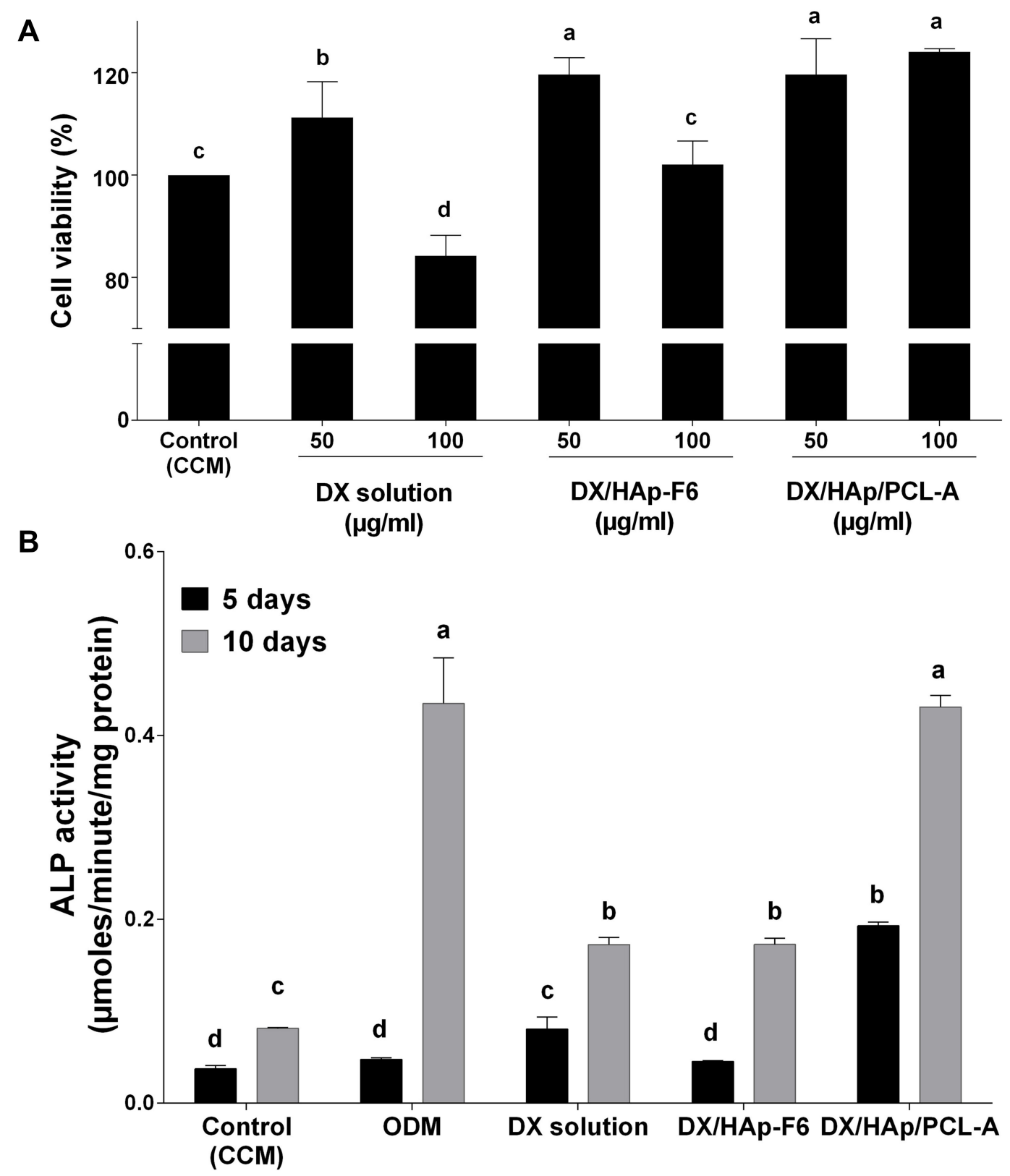

Figure 7 Cell viability and alkaline phosphatase activity of bone-marrow mesenchymal stem cells (BMSCs) in different groups (A and B). MTT cell viability of BMSCs

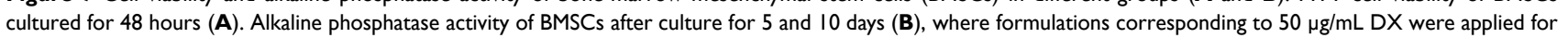
treatment groups. $n=8(\mathbf{A})$ and $n=3(\mathbf{B}) ; p \leq 0.05$; columns bearing different letters were significantly different: $a>b>c>d$.

was less pronounced than other groups. After 5-day culture, ALP activity of the DX-solution group significantly $(p \leq 0.05)$ exceeded the activity of control (CCM) and ODM groups, indicating DX osteogenic potential, in accordance with previous reports. ${ }^{8}$ Similarly to its proliferative actions, the effect of DX on osteogenic differentiation might be associated with modifications of collagen synthesis in the ECM, where a stable collagenous matrix is crucial for osteogenesis. ${ }^{18}$
The significantly $(p \leq 0.05)$ higher ALP activity of the composite DX/HAp/PCL-A group compared to other groups after culture for 5 days was expected, due to the combined effects of all its ingredients. In addition to DX osteogenic actions, HAp has been reported to increase ALP activity of human mesenchymal stem cells, ${ }^{13}$ which might be due to promoting collagen accumulation, which is necessary for osteogenesis. ${ }^{62}$ In addition, the changes exerted in culture medium as a result of incubated HApN 
might have contributed to enhanced osteogenesis. ${ }^{13}$ Further, the presence of PCL in the nanocomposite might have augmented its osteogenic potential. It has been reported that PCL-nanofiber scaffolds enhance the osteogenic potency for human mesenchymal stem cells compared to PCL films. ${ }^{63}$ The reason might be that PCL-based nanomaterial can provide favorable nanotopography that modulates the cellular microenvironment and hence directs differentiation behavior. ${ }^{65}$ After 10-day culture, the same pattern for composite DX/HAp/PCL-A could still be detected (Figure 7B). On the other hand, the long contact between BMSCs and dexamethasone in ODM might have resulted in the high ALP activity of the ODM group after 10-day culture. It has been reported that stem cells exhibit increased ALP activity after continuous 14-day exposure to dexamethasone. ${ }^{66}$

It should be mentioned that the DX-solution group was constantly replenished with fresh DX solution every time the medium was changed in the experimental setup, resulting in a cumulatively higher DX concentration than the established $50 \mu \mathrm{g} / \mathrm{mL}$. This might be one reason to explain the lower and equal ALP activity of DX/HAp-F6 group in comparison to plain DX solution after culture for 5 and 10 days, respectively. An additional reason could be the controlled-release pattern of DX from the attaching HApN matrix, offering less DX in solution than the continuously replenished free drug in the DX-solution group, unlike composite DX/HAp/PCL-A, which expressed higher initial burst release.

\section{Alizarin Red S Mineralization Assays}

During the third osteogenesis stage, the accumulated ECM undergoes mineralization. This phase involves the excessive production of bone-matrix proteins like osteocalcin and consequent aggregation of extracellular calcium deposits. ${ }^{12}$ Alizarin red S staining is a reliable technique for the evaluation of ECM mineralization. ${ }^{12}$ The dye reacts with calcium in calcified ECM, forming a chelate that can be visualized as dark-red deposits. It should be mentioned that cell monolayers of the ODM group manifested edge folding and subsequent cell clumping after culture for 7 days (data not shown) and were exempted from staining experiments. This might have resulted from the continuous 7-day exposure of BMSCs to ODM containing ascorbic acid and dexamethasone: these two ingredients have been associated with edge folding of cell sheets. ${ }^{67}$ This feature was not detected in other groups after culture for 7 days, possibly due to the adsorption of ascorbic acid and dexamethasone in HApN in other groups, with consequent controlled release minimizing that behavior. This suggestion can be supported by reports of the adsorption of ascorbic acid salt ${ }^{68}$ and dexamethasone ${ }^{69}$ from solution and into HAp and subsequent release in a controlled manner.

Microscopic examination of cells after culture for 10 days is depicted in Figure 8A. Cells in all treatment groups demonstrated morphological features probably associated with osteodifferentiation. An obvious deviation from the regular BMSCs spindle-shape morphology ${ }^{28}$ was distinguished, where cells presented a more elongated and flattened structure bearing long extensions. Also, nodular aggregates were detected, later visualized by alizarin staining as calcium deposits ${ }^{70}$ that were more pronounced for the DX/HAp-F6 and composite DX/HAp/PCL-A groups than the DX-solution group. In comparison, the control (CCM) group maintained the fibroblast-like morphology with excessive proliferation, resulting in heaped cell masses. As can be seen in Figure 8B, red deposits of mineralized nodules of various intensity were detected as early as 5 days for all treatment groups, with an overall increase in intensity after 15 -day culture. Early mineralization can be expected for cells cultured in ODM. For example, mesenchymal stem cells cultured on composite chitosan scaffolds in the presence of ODM exhibit early mineralization after 7 -day culture. ${ }^{71}$ For all time points, the DX-solution group revealed significantly $(p \leq 0.05)$ higher mineralization intensity than the control (CCM) group (Figure 8C), while the control group demonstrated an increase in mineralization intensity only after 15-day culture. Compared to other groups, however, the DX group demonstrated significantly $(p \leq 0.05)$ lower mineralization. The HAp content of those groups might have contributed to their higher mineralization intensity. Indeed, HApN possess prominent mineralization potential, probably due to the continuous release of calcium and phosphate and their subsequent deposit into an apatite layer for ECM mineralization. ${ }^{12}$ Further, calcium and phosphate ions have been reported to be essential for proliferation, differentiation, and mineralization of stem cells. ${ }^{12}$

It is clear from results in Figure $8 \mathrm{C}$ that the DX/HApF6 group presented no significant $(p \leq 0.05)$ difference in mineralization intensity compared to composite DX/HAp/ PCL-A at both 5- and 10-day time points. However, a significant $(p \leq 0.05)$ increase was detected in mineralization in the composite DX/HAp/PCL-A group at the 15-day time point. It might be that the combined osteogenic effect 
A
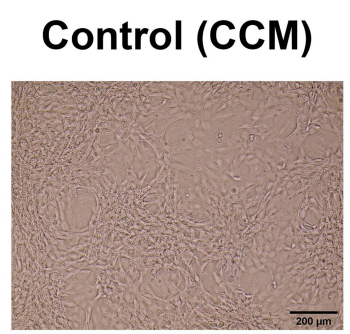

B
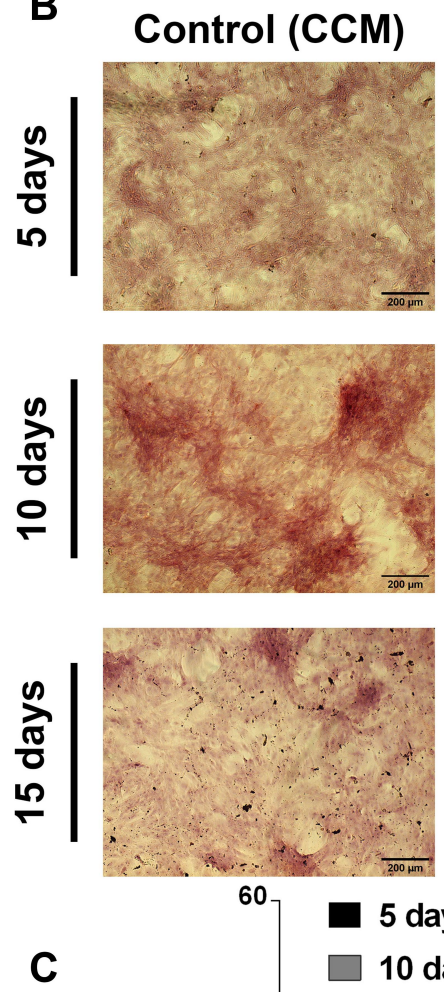

DX solution

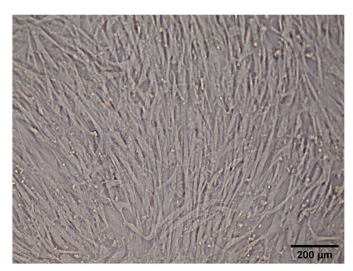

DX solution
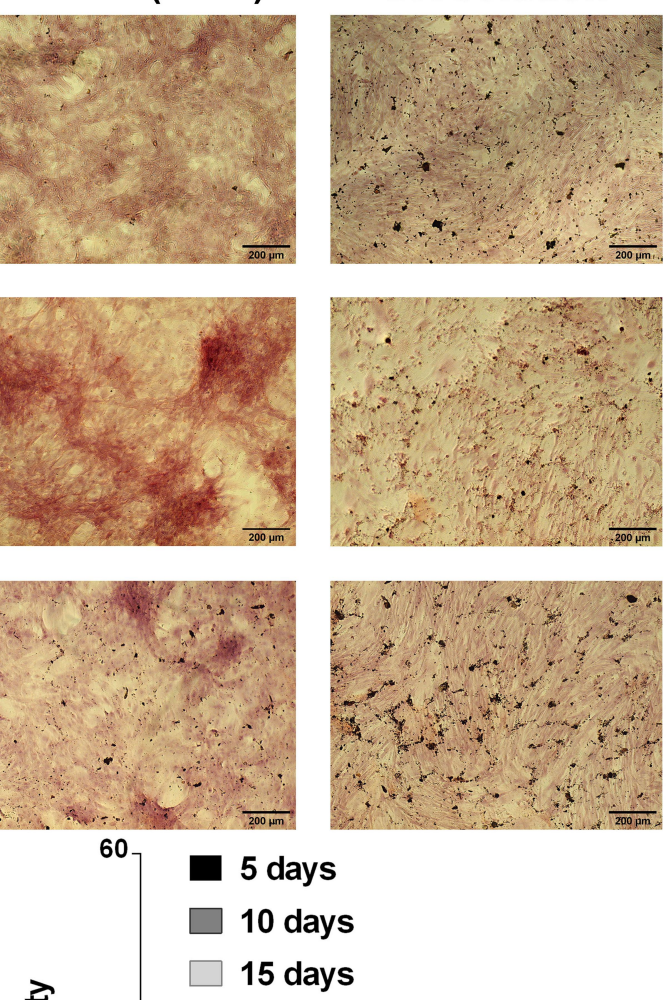

DX/HAp-F6
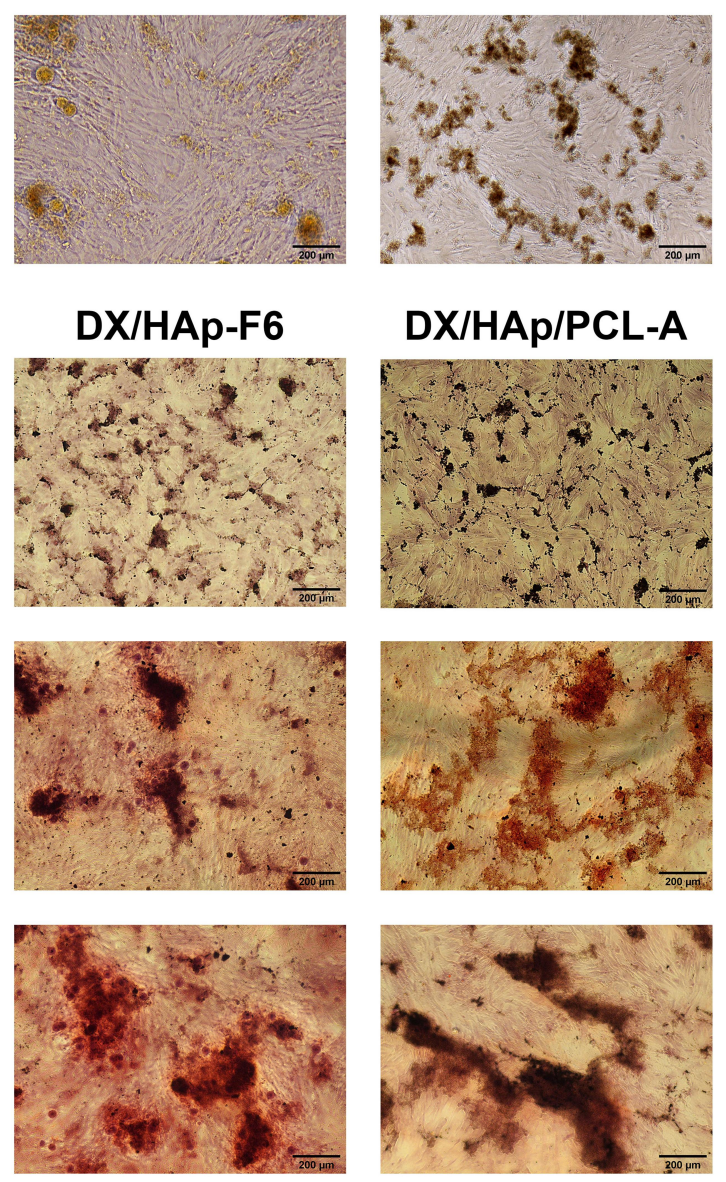

\section{DX/HAp/PCL-A}

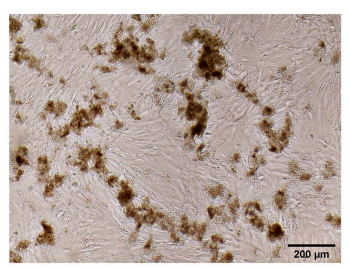

\section{DX/HAp/PCL-A}
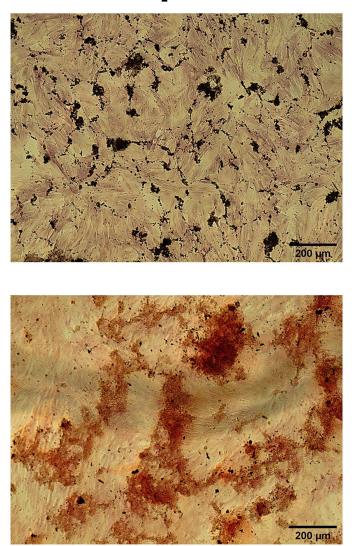

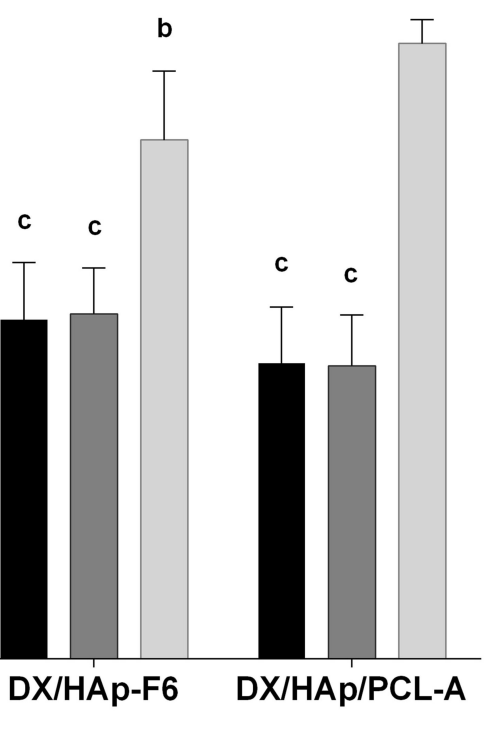

Figure 8 Alizarin red S mineralization assay for various groups (A-C), where formulations corresponding to $50 \mu g / \mathrm{mL} D X$ were applied for treatment groups. Microscopy of unstained cells for different groups after 10-day culture (A). Microscopy of stained calcium nodules for different cell groups after culture for different periods (B). Quantification of mineralized nodules $(\mathbf{C}) . n=10, p \leq 0.05$; columns bearing different letters were significantly different: $a>b>c>d>$ e $>f$. 
of all individual components resulted in superior mineralization potential of composite DX/HAp/PCL-A. In addition to HApN osteogenic actions, the polymeric PCL matrix in the nanocomposite seemingly contributed to the overall mineralization potential. It has been reported that stem cells propagated on PCL nanofibers afford uniform matrix mineralization. ${ }^{63}$ It should also be noted that the higher mineralization potential of DX/HAp/PCL-A was in accordance with the enhanced ALP activity of the composite, which confirms the overall superiority of its osteogenic activity.

\section{qRT-PCR Analysis}

Over the course of cell differentiation down the osteogenic lineage, cells exhibit upregulation and downregulation of various genes. $^{71}$ Information on gene regulation gives insight into cellular osteogenesis stages at the molecular level. To the best of our knowledge, this is the first work to elaborate the osteogenic action of DX or DX loaded particulate-delivery systems in stem cells at the molecular level using qPCR, establishing their effect on the transcription of osteogenesis-specific genes: RUNX2, OSP, and BSP.

During early osteogenesis, the transcription factor Runx2 induces osteoprogenitor proliferation and the production of bone-matrix proteins, such as ALP. ${ }^{67}$ Runx2 is first upregulated in preosteoblasts, increases in immature osteoblasts, then drops in mature osteoblasts. ${ }^{72}$ OSP is a noncollagenous phosphorylated glycoprotein that promotes cell attachment, with subsequent enhancement of mineralization. ${ }^{13}$ OSP upregulation first occurs at early-intermediate stages of osteoblast development: ${ }^{73}$ it is detected in relatively immature osteoblasts, then its expression decreases at later mineralization stages. ${ }^{74}$ Another phosphorylated glycoprotein is BSP, which stimulates initial mineralization phases of boneconnective tissue, probably via nucleation of HAp. ${ }^{75}$ BSP expression is detected in extensively differentiated osteoblasts at late maturation stages. ${ }^{76}$

As illustrated in Figure $9 \mathrm{~A}-\mathrm{C}$, all treatment groups exhibited an upregulation of all studied genes compared to the control (CCM) group, establishing their overall osteodifferentiated state. The DX-solution group demonstrated significantly $(p \leq 0.05)$ higher Runx2 and OSP expression than other groups. These results, combined with the lack of BSP expression (Figure 9C), suggest that cells in the DX-solution group were mostly immature osteoblasts after culture for 10 days, which justifies their poor mineralization manifested in the alizarin assay. In other words, DX osteogenic potential might have initiated mineralization and guided osteoprogenitor cells only until early osteodifferentiation, even though the experimental setup involved constant DX replenishment. Runx2 expression for the ODM group was slightly higher than the control (CCM) group. Judging by the low OSP and almost no BSP expression, it might be that cells in ODM were mostly preosteoblasts after 10 -day culture. ${ }^{72}$ Therefore, the high ALP activity for the ODM group after culture for 10 days might be attributed to the fact that the enzyme can be produced by differentiated cells other than osteoblasts. $^{64}$

As plotted in Figure 9A and B, both DX/HAp-F6 and composite DX/HAp/PCL-A groups manifested equally moderate Runx2 and OSP expression, while both groups revealed significantly $(p \leq 0.05)$ elevated expression of BSP (Figure 9C). This expression pattern conforms with cells undergoing intermediate- to latestage osteodifferentiation. ${ }^{72}$ Although the DX/HAp-F6 group presented higher BSP-fold change than composite DX/HAp/PCL-A group, the latter manifested higher mineralization intensity after 15-day culture, as demonstrated by alizarin assays. It could be that the composite group had higher cell mineralization and maturation later than 10-day culture, when PCR was carried out.

\section{Conclusion}

In the current study, we managed to repurpose low-dose DX using particulate nanosystems - DX/HAp and composite DX/HAp/PCL — that provided controlled release of $\mathrm{DX}$ in different patterns in order to ameliorate its dose-dependent cytotoxicity, as well as augment its osteogenic potential. The developed systems combined the complementary osteogenicity of their constituting biomaterials: HApN, PCL, and low-dose repurposed DX. Compared to low-dose DX solution alone, the novel nanocomposite $\mathrm{DX} / \mathrm{HAp} / \mathrm{PCL}$, and to a relatively less extent DX/HAp, diminished DX cytotoxicity and enhanced proliferation, osteodifferentiation, mineralization, and maturation of BMSCs during the study period. This is the first reported study, to the best of our knowledge, to optimize HApN for surface area and consequent DX loading. Also, to the best of our knowledge, there is no other previous report of DX being investigated for its effect on Runx2, OSP, or BSP expression in vitro. Further, the osteogenicity of DX-loaded particulatedelivery systems has not been previously investigated in vitro. The prominent osteogenic potential of the novel 


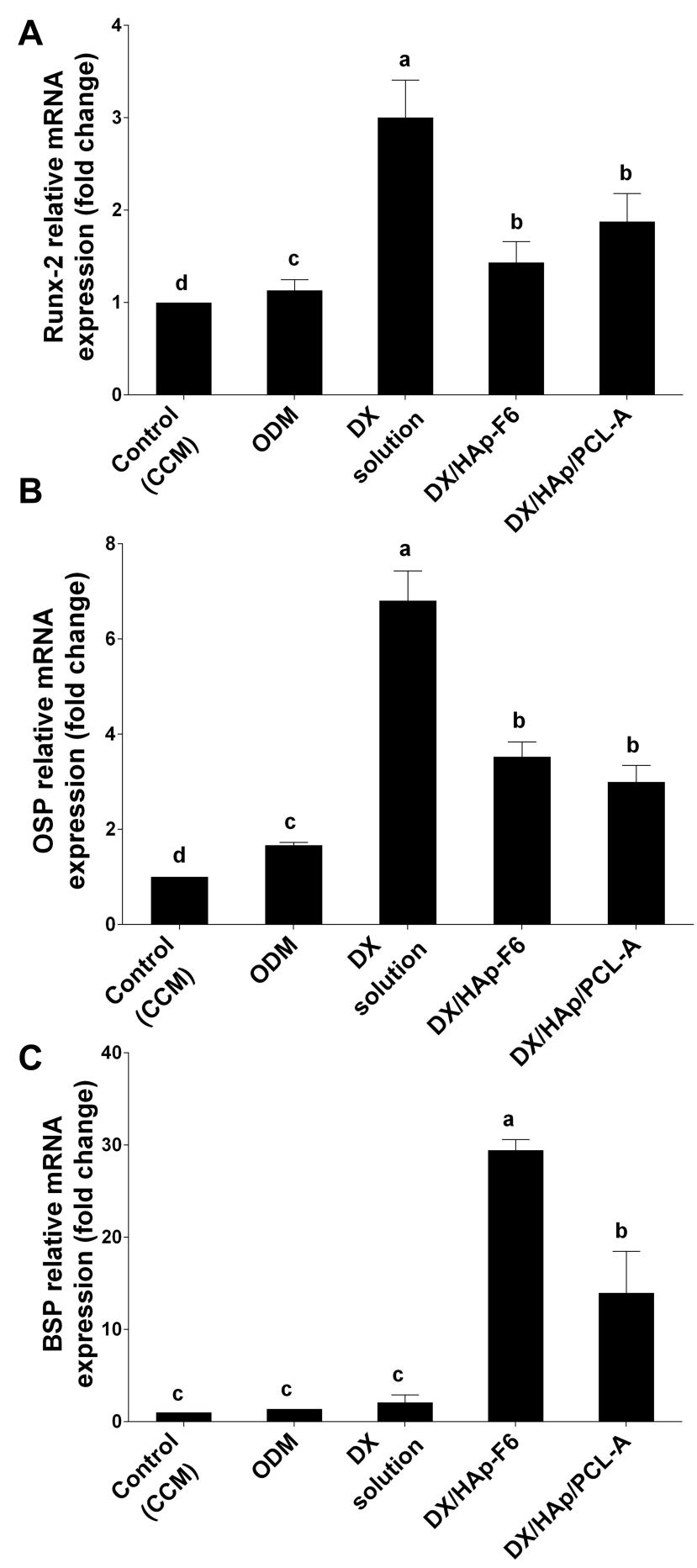

Figure 9 Enhanced expression level of osteogenic genes (A-C), where formulations corresponding to $50 \mu \mathrm{g} / \mathrm{mL} \mathrm{DX}$ were applied for treatment groups. Runx2 (A), OSP (B), and BSP (C) for different formulations after 10-day culture. $n=3$, $p \leq 0.05$; columns bearing different letters are significantly different: $a>b>c>d$.

nanocomposite $\mathrm{DX} / \mathrm{HAp} / \mathrm{PCL}$ renders it a promising candidate for further bone-regeneration purposes in vivo. It could be either directly applied as filling material or further employed to design biomimetic scaffolds for personalized bone regeneration.

\section{Data-Sharing Statement}

The authors confirm that the data for the study findings within the article are available for use.

\section{Funding}

Cell culture studies in this work were supported by the Academic Thesis Research Fund (ATRF) committee, Faculty of Pharmacy, Alexandria University (grant ATRF-0218005). The authors would like to state that the funding source was not involved in any part of the study design or manuscript preparation.

\section{Disclosure}

The authors confirm that there are no conflicts of interest associated with this work.

\section{References}

1. Rezaei A, Mohammadi MR. In vitro study of hydroxyapatite/polycaprolactone (HA/PCL) nanocomposite synthesized by an in situ solgel process. Mater Sci Eng C. 2013;33(1):390-396. doi:10.1016/j. msec.2012.09.004

2. Soriano-Souza C, Valiense H, Mavropoulos E, et al. Doxycycline containing hydroxyapatite ceramic microspheres as a bone-targeting drug delivery system. $J$ Biomed Mater Res Part B Appl Biomater. 2020;108(4):1351-1362. doi:10.1002/jbm.b.34484

3. Aravamudhan A, Ramos M, Nip J, et al. Osteoinductive small molecules: growth factor alternatives for bone tissue engineering. Curr Pharm Des. 2013;19(19):3420-3428. doi:10.2174/ 1381612811319190008

4. Carbone EJ, Rajpura K, Jiang T, Laurencin CT, Lo KWH. Regulation of bone regeneration with approved small molecule compounds. $A d v$ Regenerat Biol. 2014;1(1):25276. doi:10.3402/arb.v1.25276

5. Han -Q-Q, Du Y, Yang P-S. The role of small molecules in bone regeneration. Future Med Chem. 2013;5(14):1671-1684. doi:10.4155/fmc. 13.133

6. Eglence A, Colterjohn N, Cm Duivenvoorden W, Ghert M, Singh G. Effect of bone morphogenetic protein-2 and doxycycline on the differentiation of osteoprogenitors from human femoral bone. Open Bone J. 2009;1(1):1-7. doi:10.2174/1876525400901010001

7. Holmes SG, Still K, Buttle DJ, Bishop NJ, Grabowski PS. Chemically modified tetracyclines act through multiple mechanisms directly on osteoclast precursors. Bone. 2004;35(2):471-478. doi:10.1016/j.bone.2004.02.028

8. Park J-B. Low dose of doxycyline promotes early differentiation of preosteoblasts by partially regulating the expression of estrogen receptors. J Surg Res. 2012;178(2):737-742. doi:10.1016/j. jss.2012.03.072

9. Gomes PS, Fernandes MH. Effect of therapeutic levels of doxycycline and minocycline in the proliferation and differentiation of human bone marrow osteoblastic cells. Arch Oral Biol. 2007;52 (3):251-259. doi:10.1016/j.archoralbio.2006.10.005

10. Park J-B. Effects of doxycycline, minocycline, and tetracycline on cell proliferation, differentiation, and protein expression in osteoprecursor cells. J Craniofac Surg. 2011;22(5):1839-1842. doi:10.1097/ SCS.0b013e 31822e 8216

11. Nawang R, Hussein MZ, Matori KA, Abdullah CAC, Hashim M. Physicochemical properties of hydroxyapatite/montmorillonite nanocomposite prepared by powder sintering. Results Phys. 2019;15:102540. doi:10.1016/j.rinp.2019.102540 
12. Kazimierczak P, Kolmas J, Przekora A. Biological response to macroporous chitosan-agarose bone scaffolds comprising Mg-and Zn-doped nano-hydroxyapatite. Int J Mol Sci. 2019;20(15):3835. doi:10.3390/ijms20153835

13. Yang X, Li Y, Liu X, Zhang R, Feng Q. In vitro uptake of hydroxyapatite nanoparticles and their effect on osteogenic differentiation of human mesenchymal stem cells. Stem Cells Int. 2018;2018:2018. doi:10.1155/2018/2036176

14. Ding L, Zhang P, Wang X, et al. Effect of doxycycline-treated hydroxyapatite surface on bone apposition: a histomophometric study in murine maxillae. Dent Mater J. 2018;37(1):130-138. doi:10.4012/dmj.2017-007

15. Ramírez-Agudelo R, Scheuermann K, Gala-García A, et al. Hybrid nanofibers based on poly-caprolactone/gelatin/hydroxyapatite nanoparticles-loaded Doxycycline: effective anti-tumoral and antibacterial activity. Mater Sci Eng C. 2018;83:25-34. doi:10.1016/j. msec.2017.08.012

16. Kamath MS, Ahmed SSSJ, Dhanasekaran M, Santosh SW. Polycaprolactone scaffold engineered for sustained release of resveratrol: therapeutic enhancement in bone tissue engineering. Int $J$ Nanomedicine. 2014;9:183. doi:10.2147/IJN.S49460

17. Woodruff MA, Hutmacher DW. The return of a forgotten polymerPolycaprolactone in the 21st century. Prog Polym Sci. 2010;35 (10):1217-1256. doi:10.1016/j.progpolymsci.2010.04.002

18. Gomes PS, Santos JD, Fernandes MH. Cell-induced response by tetracyclines on human bone marrow colonized hydroxyapatite and Bonelike $^{\circledR}$. Acta Biomater. 2008;4(3):630-637. doi:10.1016/j. actbio.2007.12.006

19. El-Habashy SE, Eltaher HM, Gaballah A, Zaki EI, Mehanna RA, ElKamel AH. Hybrid bioactive hydroxyapatite/polycaprolactone nanoparticles for enhanced osteogenesis. Mater Sci Eng C. 2021;119:111599. doi:10.1016/j.msec.2020.111599

20. Manoj M, Subbiah R, Mangalaraj D, Ponpandian N, Viswanathan C, Park K. Influence of growth parameters on the formation of Hydroxyapatite (HAp) nanostructures and their cell viability studies. Nanobiomedicine (Rij). 2015;2:2. doi:10.5772/60116

21. Sadat-Shojai M, Khorasani MT, Dinpanah-Khoshdargi E, Jamshidi A. Synthesis methods for nanosized hydroxyapatite with diverse structures. Acta Biomater. 2013;9(8):7591-7621.

22. Madhumathi K, Sampath Kumar TS. Regenerative potential and antibacterial activity of tetracycline loaded apatitic nanocarriers for the treatment of periodontitis. Biomed Mater. 2014;9(3):035002. doi:10.1088/1748-6041/9/3/035002

23. Wang X, Xu H, Zhao Y, et al. Poly(lactide-co-glycolide) encapsulated hydroxyapatite microspheres for sustained release of doxycycline. Mat Sci Eng B Adv. 2012;177(4):367-372. doi:10.1016/j. mseb.2011.12.030

24. Schindelin J, Arganda-Carreras I, Frise E, et al. Fiji: an open-source platform for biological-image analysis. Nat Methods. 2012;9(7):676682. doi:10.1038/nmeth.2019

25. Meski S, Ziani S, Khireddine H, Boudboub S, Zaidi S. Factorial design analysis for sorption of zinc on hydroxyapatite. J Hazard Mater. 2011;186(2-3):1007-1017. doi:10.1016/j. jhazmat.2010.11.087

26. Wang S, Wang $\mathrm{X}, \mathrm{Xu} \mathrm{H}$, et al. Towards sustained delivery of small molecular drugs using hydroxyapatite microspheres as the vehicle. Adv Powder Technol. 2010;21(3):268-272. doi:10.1016/j. apt.2009.12.001

27. Niu X, Liu Z, Tian F, et al. Sustained delivery of calcium and orthophosphate ions from amorphous calcium phosphate and poly (L-lactic acid)-based electrospinning nanofibrous scaffold. Sci Rep. 2017;7:45655. doi:10.1038/srep45655

28. Zhou H, Li D, Shi C, et al. Effects of Exendin-4 on bone marrow mesenchymal stem cell proliferation, migration and apoptosis in vitro. Sci Rep. 2015;5:12898. doi:10.1038/srep12898
29. Kim HK, Park KS, Lee JS, et al. Salicylideneamino-2-thiophenol enhances osteogenic differentiation through the activation of MAPK pathways in multipotent bone marrow stem cell. $J$ Cell Biochem. 2012;113(6):1833-1841. doi:10.1002/jcb.24052

30. Ernst O, Zor T. Linearization of the bradford protein assay. $J$ Vis Exp. 2010;(38):e1918. doi:10.3791/1918

31. Choudhury PR, Mondal P, Majumdar S. Synthesis of bentonite clay based hydroxyapatite nanocomposites cross-linked by glutaraldehyde and optimization by response surface methodology for lead removal from aqueous solution. RSC Adv. 2015;5(122):100838-100848. doi:10.1039/C5RA18490H

32. Xie W, Han Y, Tai S. Biodiesel production using biguanide-functionalized hydroxyapatite-encapsulated- $\gamma$-Fe2O3 nanoparticles. Fuel. 2017;210:83-90. doi:10.1016/j.fuel.2017.08.054

33. Tabassum S, Zahid S, Zarif F, et al. Efficient drug delivery system for bone repair by tuning the surface of hydroxyapatite particles. RSC Adv. 2016;6(107):104969-104978. doi:10.1039/C6RA24551J

34. Mazinani B, Beitollahi A, Masrom AK, Ibrahim S, Jamil F The effect of aging temperature on the pores of mesoporous SBA-15 silica. Paper presented at: AIP conference proceedings; 2012, Sabah, Malaysia.

35. Barabás R, Czikó M, Dékány I, Bizo L, Bogya ES. Comparative study of particle size analysis of hydroxyapatite-based nanomaterials. Chem Pap. 2013;67(11):1414-1423. doi:10.2478/s11696-0130409-6

36. Iswar S, Malfait WJ, Balog S, Winnefeld F, Lattuada M, Koebel MM. Effect of aging on silica aerogel properties. Microporous Mesoporous Mater. 2017;241:293-302. doi:10.1016/j. micromeso.2016.11.037

37. Guo Y, Cui X, Li Y, Zhang Q, Wang H. The effect of aging time on the properties of $\mathrm{Mg}-\mathrm{Al}-\mathrm{CO} 3$ layered double hydroxides and its application as a catalyst support for TiO2. J Nanosci Nanotechnol. 2016;16(6):5653-5661. doi:10.1166/jnn.2016.11725

38. Al-Qasas NS, Rohani S. Synthesis of pure hydroxyapatite and the effect of synthesis conditions on its yield, crystallinity, morphology and mean particle size. Sep Sci Technol. 2005;40(15):3187-3224. doi:10.1080/01496390500385400

39. Zanotto A, Saladino M, Chillura Martino D, Caponetti E. Influence of temperature on calcium hydroxyapatite nanopowders. $A N B$. 2012;01:21-28.

40. Kothapalli C, Wei M, Vasiliev A, Shaw MT. Influence of temperature and concentration on the sintering behavior and mechanical properties of hydroxyapatite. Acta Mater. 2004;52(19):5655-5663. doi:10.1016/j.actamat.2004.08.027

41. Huang Y-T, Imura M, Nemoto Y, Cheng C-H, Yamauchi Y. Block-copolymer-assisted synthesis of hydroxyapatite nanoparticles with high surface area and uniform size. Sci Technol Adv Mater. 2011;12(4):045005. doi:10.1088/1468-6996/12/4 /045005

42. Jafari S, Maleki-Dizaji N, Barar J, Barzegar-Jalali M, Rameshrad M, Adibkia K. Methylprednisolone acetate-loaded hydroxyapatite nanoparticles as a potential drug delivery system for treatment of rheumatoid arthritis: in vitro and in vivo evaluations. Eur $J$ Pharm Sci. 2016;91:225-235. doi:10.1016/j.ejps.2016. 05.014

43. Li Y, Wang S, Zhang Y, Han R, Wei W. Enhanced tetracycline adsorption onto hydroxyapatite by Fe (III) incorporation. J Mol Liq. 2017;247:171-181. doi:10.1016/j.molliq.2017.09.110

44. El-Habashy SE, Allam AN, El-Kamel AH. Ethyl cellulose nanoparticles as a platform to decrease ulcerogenic potential of piroxicam: formulation and in vitro/in vivo evaluation. Int $J$ Nanomedicine. 2016;11:2369. doi:10.2147/IJN.S93354

45. Misra R, Acharya S, Dilnawaz F, Sahoo SK. Sustained antibacterial activity of doxycycline-loaded poly (D, L-lactide-co-glycolide) and poly (E-caprolactone) nanoparticles. Nanomedicine. 2009;4(5):519530. doi: $10.2217 / \mathrm{nnm} .09 .28$ 
46. Mundargi RC, Srirangarajan S, Agnihotri SA, et al. Development and evaluation of novel biodegradable microspheres based on poly (d, 1lactide-co-glycolide) and poly ( $\varepsilon$-caprolactone) for controlled delivery of doxycycline in the treatment of human periodontal pocket: in vitro and in vivo studies. J Control Release. 2007;119(1):59-68. doi:10.1016/j.jconrel.2007.01.008

47. Raval JP, Naik DR, Amin KA, Patel PS. Controlled-release and antibacterial studies of doxycycline-loaded poly ( $\varepsilon$-caprolactone) microspheres. J Saudi Chem Soc. 2014;18(5):566-573. doi:10.1016/ j.jscs.2011.11.004

48. Hu Q, Tan Z, Liu Y, et al. Effect of crystallinity of calcium phosphate nanoparticles on adhesion, proliferation, and differentiation of bone marrow mesenchymal stem cells. J Mater Chem. 2007;17(44):4690 4698. doi:10.1039/b710936a

49. He Z-X, Wang Z-H, Zhang H-H, et al. Doxycycline and hydroxypropyl- $\beta$-cyclodextrin complex in poloxamer thermal sensitive hydrogel for ophthalmic delivery. Acta Pharm Sin B. 2011;1(4):254-260. doi:10.1016/j.apsb.2011.10.004

50. Alshora DH, Ibrahim MA, Alanazi FK. Nanotechnology from particle size reduction to enhancing aqueous solubility. Surf Chem Nanobiomater. 2016;163-191.

51. Farah S, Doloff JC, Müller P, et al. Long-term implant fibrosis prevention in rodents and non-human primates using crystallized drug formulations. Nat Mater. 2019;18(8):892. doi:10.1038/s41563019-0377-5

52. Ignjatović $\mathrm{N}$, Uskoković $\mathrm{V}$, Ajduković $\mathrm{Z}$, Uskoković D. Multifunctional hydroxyapatite and poly (D, L-lactide-co-glycolide) nanoparticles for the local delivery of cholecalciferol. Mater Sci Eng C. 2013;33(2):943-950. doi:10.1016/j.msec.2012.11.026

53. Yadav AA, Masumdar EU. Preparation and characterization of indium doped $\mathrm{CdS} 0.2 \mathrm{Se} 0.8$ thin films by spray pyrolysis. Mater Res Bull. 2010;45(10):1455-1459. doi:10.1016/j. materresbull.2010.06.034

54. Tshweu L, Katata L, Kalombo L, et al. Enhanced oral bioavailability of the antiretroviral efavirenz encapsulated in poly (epsilon-caprolactone) nanoparticles by a spray-drying method. Nanomedicine. 2014;9 (12):1821-1833. doi:10.2217/nnm.13.167

55. Banik M, Basu T. Calcium phosphate nanoparticles: a study of their synthesis, characterization and mode of interaction with salmon testis DNA. Dalton Trans. 2014;43(8):3244-3259. doi:10.1039/ C3DT52522H

56. Dorozhkin SV. Nanodimensional and nanocrystalline apatites and other calcium orthophosphates in biomedical engineering, biology and medicine. Materials (Basel). 2009;2(4):1975-2045.

57. Salimi MN, Bridson RH, Grover LM, Leeke GA. Effect of processing conditions on the formation of hydroxyapatite nanoparticles. Powder Technol. 2012;218:109-118. doi:10.1016/j. powtec.2011.11.049

58. Shanthi PMSL, Mangalaraja RV, Uthirakumar AP, Velmathi S, Balasubramanian T, Ashok M. Synthesis and characterization of porous shell-like nano hydroxyapatite using Cetrimide as template. $J$ Colloid Interface Sci. 2010;350(1):39-43. doi:10.1016/j. jcis.2010.05.046

59. Borjigin M, Eskridge C, Niamat R, Strouse B, Bialk P, Kmiec EB. Electrospun fiber membranes enable proliferation of genetically modified cells. Int J Nanomedicine. 2013;8:855. doi:10.2147/IJN.S40117

60. Chan KH, Zhuo S, Ni M. Priming the surface of orthopedic implants for osteoblast attachment in bone tissue engineering. Int J Med Sci. 2015;12(9):701-707. doi:10.7150/ijms.12658

61. Zhang L, Xu L, Zhang F, Vlashi E. Doxycycline inhibits the cancer stem cell phenotype and epithelial-to-mesenchymal transition in breast cancer. Cell Cycle. 2017;16(8):737-745. doi:10.1080/ 15384101.2016.1241929
62. Liu Y, Wang G, Cai Y, et al. In vitro effects of nanophase hydroxyapatite particles on proliferation and osteogenic differentiation of bone marrow-derived mesenchymal stem cells. J Biomed Mater Res A. 2009;90A(4):1083-1091. doi:10.1002/jbm.a.32192

63. Xue R, Qian Y, Li L, Yao G, Yang L, Sun Y. Polycaprolactone nanofiber scaffold enhances the osteogenic differentiation potency of various human tissue-derived mesenchymal stem cells. Stem Cell Res Ther. 2017;8(1):148. doi:10.1186/s13287-017-0588-0

64. Polini A, Pisignano D, Parodi M, Quarto R, Scaglione S, Gimble JM. Osteoinduction of human mesenchymal stem cells by bioactive composite scaffolds without supplemental osteogenic growth factors. PLoS One. 2011;6(10):e26211. doi:10.1371/journal.pone.0026211

65. Liu D, Yi C, Fong -C-C, et al. Activation of multiple signaling pathways during the differentiation of mesenchymal stem cells cultured in a silicon nanowire microenvironment. Nanomedicine. 2014;10(6):1153-1163. doi:10.1016/j.nano.2014.02.003

66. Oliveira JM, Sousa RA, Kotobuki N, et al. The osteogenic differentiation of rat bone marrow stromal cells cultured with dexamethasone-loaded carboxymethylchitosan/poly (amidoamine) dendrimer nanoparticles. Biomaterials. 2009;30(5):804-813. doi:10.1016/j. biomaterials.2008.10.024

67. Kim AY, Kim Y, Lee SH, Yoon Y, Kim W-H, Kweon O-K. Effect of gelatin on osteogenic cell sheet formation using canine adiposederived mesenchymal stem cells. Cell Transplant. 2017;26(1):115123. doi:10.3727/096368916X693338

68. Zhu Y, Liu J, Zhu W, Cai Y, Kundu SC, Yao J. Surface modification of hydroxyapatite microspheres for the sustained release of vitamin C. Mater Technol. 2015;30(sup5):B223-B228. doi:10.1179/ 17535557B15Y.000000007

69. Tavakoli-Darestani R, Manafi-Rasi A, Kamrani-Rad A. Dexamethasone-loaded hydroxyapatite enhances bone regeneration in rat calvarial defects. Mol Biol Rep. 2014;41(1):423-428. doi:10.1007/s11033-013-2876-9

70. Georgopoulou A, Kaliva M, Vamvakaki M, Chatzinikolaidou M. Osteogenic potential of pre-osteoblastic cells on a chitosan-graftpolycaprolactone copolymer. Materials (Basel). 2018;11(4):490. doi: $10.3390 /$ ma11040490

71. Lai G-J, Shalumon KT, Chen J-P. Response of human mesenchymal stem cells to intrafibrillar nanohydroxyapatite content and extrafibrillar nanohydroxyapatite in biomimetic chitosan/silk fibroin/nanohydroxyapatite nanofibrous membrane scaffolds. Int J Nanomedicine. 2015;10:567. doi:10.2147/IJN.S73780

72. Lorenzo J, Horowitz M, Choi Y, Takayanagi H, Schett G, Eds. Osteoimmunology: Interactions of the Immune and Skeletal Systems II. US: Springer; 2009.

73. Yamaguchi Y, Ohno J, Sato A, Kido H, Fukushima T. Mesenchymal stem cell spheroids exhibit enhanced in-vitro and in-vivo osteoregenerative potential. BMC Biotechnol. 2014;14(1):105. doi:10.1186/ s12896-014-0105-9

74. Deegan AJ, Aydin HM, Hu B, Konduru S, Kuiper JH, Yang Y. A facile in vitro model to study rapid mineralization in bone tissues. Biomed Eng Online. 2014;13(1):136. doi:10.1186/1475-925X-13-136

75. Tang J, Saito T. Human plasma fibronectin promotes proliferation and differentiation of odontoblast. J Appl Oral Sci. 2017;25(3):299-309. doi:10.1590/1678-7757-2016-0442

76. Wang F, Okawa H, Kamano Y, et al. Controlled osteogenic differentiation of mouse mesenchymal stem cells by tetracycline-controlled transcriptional activation of amelogenin. PLoS One. 2015;10(12): e0145677. doi:10.1371/journal.pone.0145677 


\section{Publish your work in this journal}

The International Journal of Nanomedicine is an international, peerreviewed journal focusing on the application of nanotechnology in diagnostics, therapeutics, and drug delivery systems throughout the biomedical field. This journal is indexed on PubMed Central, MedLine, CAS, SciSearch ${ }^{\mathbb{R}}$, Current Contents ${ }^{\mathbb{B}} /$ Clinical Medicine,
Journal Citation Reports/Science Edition, EMBase, Scopus and the Elsevier Bibliographic databases. The manuscript management system is completely online and includes a very quick and fair peer-review system, which is all easy to use. Visit http://www.dovepress.com/ testimonials.php to read real quotes from published authors. 